\title{
Improving communication between parents and adolescents on reproductive health and HIV/AIDS
}

Nafissatou J. Diop

Population Council

Alioune Diagne

Follow this and additional works at: https://knowledgecommons.popcouncil.org/departments_sbsr-rh

Part of the Demography, Population, and Ecology Commons, International Public Health Commons, and the Public Health Education and Promotion Commons How does access to this work benefit you? Let us know!

\section{Recommended Citation}

Diop, Nafissatou J. and Alioune Diagne. 2008. "Improving communication between parents and adolescents on reproductive health and HIV/AIDS," FRONTIERS Final Report. Washington, DC: Population Council. 


\title{
Improving communication between parents and adolescents on reproductive health and HIV/AIDS
}

\author{
Frontiers in Reproductive Health, Population Council \\ Nafissatou J. Diop, Ph.D \\ Alioune Diagne, Ph.D
}

March 2008

This study was made possible by the generous support of the American people through the United States Agency for International Development (USAID) under the terms of Cooperative Agreement No. HRN-A00-98-00012-00. The contents are the responsibility of the FRONTIERS Program and do not necessarily reflect the views of USAID or the United States Government.
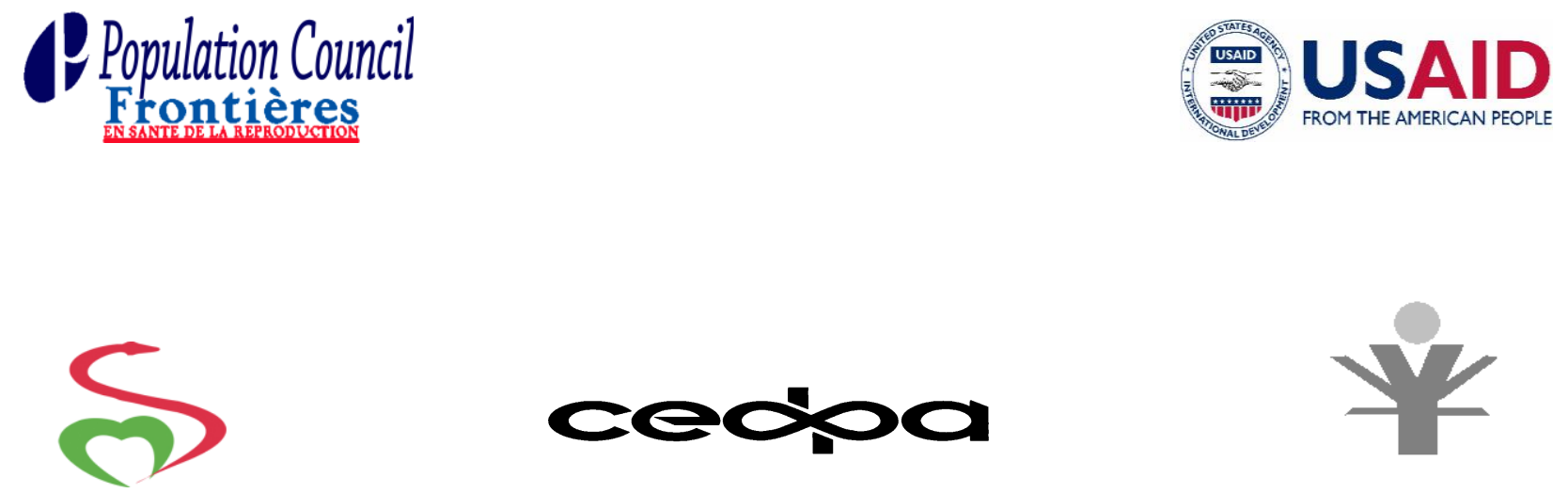


\section{EXECUTIVE SUMMARY}

In 2005 the Population Council's FRONTIERS in Reproductive Health Program, in collaboration with the Centre for Development and Population Activities (CEDPA), the Ministry of Health and the Ministry of Youth in Senegal, conducted an operations research project aimed at improving parent-child (specifically parent-adolescent) communication about reproductive health and HIV/AIDS. The study was designed to focus on parents/guardians of adolescents (1014 years and 15-19 years) and adolescent youth living in urban and rural Senegal. The overall objective was to gain a better understanding of interventions designed specifically to reduce risks to Adolescent Reproductive Health.

The specific goal of the project was to develop a model of intervention and assess its effectiveness and functionality in increasing adult-youth communication around issues of adolescent well-being, sexuality and reproductive health in terms of changes in the areas of:

- Parents' and youth's knowledge and attitudes about issues concerning adolescents, particularly reproductive health;

- Community support for adolescent needs, particularly in communication on adolescents' reproductive health issues.

- The frequency, quality, and topics discussed during parent-child (parent-adolescent) communication;

- The protective and regulatory mechanisms used by parents to address adolescents' reproductive health.

This subject is very relevant given the importance of parental-child communication in the healthy development of children, particularly as it concerns sexual development and reproductive health. However, in the African context, literature on parent-adolescent communication is scarce. Only a few diagnostic studies recognizing the importance and willingness of establishing this communication are available. In the African context, adolescents learn most of what they know about sex and reproductive health from friends and media sources (Pacific Institute for Women's Health 2002; Dieng et al. 2001). Indeed, the link between parent-child communication and sexual behavior has only begun to be explored.

A study conducted in Senegal (CEFFEVA, 2001) found that adolescents and their parents view communicating about sex differently. Adolescents say discussions focus on the consequences of premarital sex rather than more specific information about preventing pregnancy and disease. Parents believe that providing such information may actually encourage sexual activity. Moreover, parents are reluctant to talk to their children about prevention because they don't feel adequately informed to do so (Dieng et al. 2001). Nearly all the parents surveyed rated their knowledge about sexually transmitted diseases (STDs) and pregnancy prevention as inadequate. The study also revealed that parents are more likely to communicate with girls about sex than with boys, and when they did, the discussion was about pregnancy.

In general, the research showed that communication between Senegalese parents and adolescents about sex, when it occurred, was one-sided with parents admonishing adolescents rather than listening to their concerns or answering their questions. Despite the difficulties involved in communication about sexuality, Senegalese parents recognize that adolescents need information 
about reproductive health and want to be the main actors in educating their children about these issues (Dieng et al., 2001; Diop et al., 2004). Youth say they would prefer to obtain information from their parents about sexual matters than from peers or others (Dieng et al. 2001). The desire for intergenerational communication is reciprocal. In the study reported here, $75 \%$ of parents said they would like to give information to their children.

This type of communication is not easy. After FRONTIERS implemented a set of interventions designed to improve adolescent reproductive health, the youths involved in the interventions reported seeking more information from adult mentors rather than their friends, but they did not report having better communication with their parents (Diop et al, 2004).

\section{Methodology}

The research was conducted in three phases:

- Phase I: Pre-test/baseline assessment of the initial situation;

- Phase II: Intervention activities led by CEDPA in the communities selected as intervention zones;

- Phase III: Final post-test evaluation assessment.

The study used a panel design with a comparison area. The units of analysis were adolescents and adults in the community who participate in the intervention:

$\begin{array}{llll}\text { Experimental Group } & \mathrm{O}_{1} & \mathrm{X} & \mathrm{O}_{2} \\ \text { Comparison Group } & \mathrm{O}_{3} & & \mathrm{O}_{4}\end{array}$

Where:

$\mathbf{O}_{1}$ and $\mathbf{O}_{3}=$ Pretest survey to measure adult and youth knowledge, attitudes, and behavior concerning reproductive health and intergenerational communication. The pretest survey was carried out after the advocacy activities in the experimental area and comparison area.

$\mathbf{X}=$ Intervention activities

$\mathbf{O}_{2}$ and $\mathbf{O}_{4}=$ Post-test survey to measure adult and youth knowledge, attitudes, behavior concerning reproductive health and intergenerational communication. The posttest survey was conducted 10 months after the intervention activities had been implemented in the experimental and comparison area.

A Baseline Survey was conducted prior to introduction of the intervention activities. The objective of this first step was to gather sufficient information about the existing situation concerning the frequency and quality of parent-child communications about RH/HIV/AIDS issues. Questionnaires were created specifically for youths and parents/guardians. Each question was linked to a particular aspect or issue addressed in the study.

This was followed by implementation of the intervention activities. The goal of the intervention activities was to help build the capacity of the adolescents and their parent/guardians and improve the level and quality of the communication between them about RH issues. The activities consisted of advocacy and sensitivity training sessions for youths and their 
parents/guardians. The activities were conducted for four months (September 2005-December 2006).

Implementation of the intervention was followed by qualitative investigations that, using a socio-anthropological approach, were designed to gain a better understanding of the channels used by Senegalese families to address the need to provide information about adolescent reproductive health. This survey also helped identify the mechanisms of parent-child RH communication and the environment in which such communication takes place. These qualitative data was gathered through focus groups and personal testimonies.

The final post-test phase of the study was an evaluation. It was conducted in December 2006 12 months after the final intervention activity. This phase focused on identifying the changes that had taken place in the community concerning parent/guardian-adolescents RH communication, specifically looking at the impact of the interventions on parent-adolescent communication and also examining whether they contributed to the emergence of any new practices and behaviors in the area of intergenerational communication.

At every step of the data collection process, the research team adhered to ethical research principles. Participation in this study was voluntary. People were informed that no one was required to participate if he/she felt uncomfortable doing so. Informed Consent was obtained from each participant. Participants were fully informed of the purpose of the study and guaranteed that all information obtained would be treated confidentially and anonymously.

A total of 1,293 adolescents (male and female) and 896 parent/guardians (all randomly selected at the household level) were interviewed for the baseline study. For the final survey, a total of 1,160 adolescents and 762 parents/guardians interviewed; among these, 91\% of the youth and their parents were the same as baseline (See Table 1).

Table No. 1: Summary of sample sizes

\begin{tabular}{|l|cc|cc|cc|}
\hline & \multicolumn{2}{c|}{ Urban } & \multicolumn{2}{c|}{ Rural } & \multicolumn{2}{c|}{ Total } \\
\cline { 2 - 8 } & $\begin{array}{c}\text { Baseline } \\
\text { Survey }\end{array}$ & $\begin{array}{c}\text { Final } \\
\text { Survey }\end{array}$ & $\begin{array}{c}\text { Baseline } \\
\text { Survey }\end{array}$ & $\begin{array}{c}\text { Final } \\
\text { Survey }\end{array}$ & $\begin{array}{c}\text { Baseline } \\
\text { Survey }\end{array}$ & $\begin{array}{c}\text { Final } \\
\text { Survey }\end{array}$ \\
\hline Boys & 29 & 22 & 98 & 38 & 127 & 60 \\
\hline $10-14$ years old & 115 & 75 & 279 & 116 & 394 & 191 \\
$15-19$ years old & 47 & 150 & 142 & 205 & 189 & 355 \\
\hline Girls & 182 & 224 & 401 & 330 & 583 & 554 \\
\hline $10-14$ years old & \multicolumn{7}{|c|}{} \\
15-19 years old & 373 & 471 & 920 & 689 & 1293 & 1160 \\
\hline Total & $\mathbf{2 6 2}$ & $\mathbf{1 7 2}$ & $\mathbf{6 4 4}$ & $\mathbf{5 9 0}$ & $\mathbf{9 0 6}$ & $\mathbf{7 6 2}$ \\
\hline Adolescents
\end{tabular}

Note: There was a slight decrease in the response rate from youths and adults in the final survey. Among the adolescents this can be attributed to the timing of the surveys. The baseline survey was conducted during the school vacation break and so the majority of the respondents were students. The final survey was conducted during the school year, often during daytime school hours when it was difficult to find the respondents. Also, some of the adolescents had moved away to pursue their studies in another town. 


\section{Institutional Framework}

A multi-sectoral approach was adopted to allow for the participation of several stakeholders working in the area of reproductive health. CEDPA (the Centre for Development and Population Activities) was the lead organization for implementing the intervention activities. The Population Council's FRONTIERS in Reproductive Health Program was responsible for the research component and process documentation, including the baseline study and the evaluation. USAID supported both components through separate contracts with each organization. CEDPA and FRONTIERS worked in close collaboration with Senegal's Ministry of Health and Preventive Medicine (mostly through the Division of Reproductive Health and the medical region of Kaolack) and the Ministry of Youth (Youth and Associative Life Division). Also collaborating on this project were several organizations working in the area of reproductive health, among them: USAID, WHO, UNFPA, UNICEF, PPJ, ADEMAS and GEEP, etc. Periodic meetings were held to inform and assure common comprehension about the organizational roles and duties, and progress of the project. The discussions and negotiations that took place during those meetings brought out critical aspects of the project and helped bring about a favorable appropriation and ownership of the results by all the stakeholders.

\begin{tabular}{|c|c|c|}
\hline TECHNICAL COMMITTEE & $\begin{array}{l}\Rightarrow \\
\Rightarrow \\
\Rightarrow \\
\Rightarrow \\
\Rightarrow \\
\Rightarrow \\
\Rightarrow \\
\Rightarrow\end{array}$ & $\begin{array}{l}\text { DRH (Ministry of Health, Department of Reproductive Health) } \\
\text { DJVA (Ministry of Youth, Youth and Associative Life Division) } \\
\text { PPJ (Ministry of Youth, Promotion of Youth Project) } \\
\text { Population Council /FRONTIERS } \\
\text { CEDPA /BRIDGE } \\
\text { USAID } \\
\text { WHO } \\
\text { UNICEF }\end{array}$ \\
\hline $\begin{array}{c}\text { DEPARTMENTAL STEERING } \\
\text { COMMITTEE }\end{array}$ & $\begin{array}{l}\Rightarrow \\
\Rightarrow \\
\Rightarrow \\
\Rightarrow \\
\Rightarrow \\
\Rightarrow\end{array}$ & $\begin{array}{l}\text { District Chief, Medical District of Kaolack } \\
\text { Inspector, Department of Youth } \\
\text { Head of Service, Department of Community Development } \\
\text { Reproductive Health Promotion Network } \\
\text { NGOS targeting women/youth } \\
\text { Association Members }\end{array}$ \\
\hline LOCAL STEERING COMMITTEE & $\begin{array}{l}\Rightarrow \\
\Rightarrow \\
\Rightarrow \\
\Rightarrow\end{array}$ & $\begin{array}{l}\text { ICP (Head Post Nurse) } \\
\text { Parents/Adults Peer Educators } \\
\text { Youth Peer Educators } \\
\text { Moderators } \\
\text { Village Associations, etc. }\end{array}$ \\
\hline
\end{tabular}

\section{Intergenerational Communication: Life Skills Sessions}

The key component of this intervention was the establishment of intergenerational dialogues between young people and adults. It was originally planned that both adult Peer Educators and Youth Peer Educators would organize dialogue sessions every month. The Reproductive Health Promotion Network (RPSR), a CEDPA partner for youth activities in the Kaolack region, was charged with implementing the various intervention activities with the assistance of CEDPAtrained Peer Educators (20 adults and 20 youth). There were 10 supervisors for each of the five target study sites: one urban and four rural in the Department of Kaolack. 
Each peer educator worked with four groups of 20 adolescents and organized five Life Skills classes per week. These were held after school. The topics covered were: Learning to Communicate with Parents; Learning to Understand Ourselves; Exploring Our Expectations; Resisting Peer Pressure and Making Good Decisions. A total of 1,528 adolescents participated in the Life Skills training sessions.

Discussion sessions for adults (parents/guardians, both male and female) were also organized. Each adult facilitator worked with four groups of parents/guardians. Five sessions per week were held over a period of five months. Topics discussed were: Helping Children Prevent Pregnancy; Helping Children Protect Themselves against STI/HIV/AIDS, and Supporting Children's Choices. A total of 1,512 parents/guardians participated in these sessions.

In addition, at each site, at the end of each discussion session, an Intergenerational Dialogue Session was organized in which discussions focused on specific RH topics. The purpose of these sessions was to bring parents/guardians and their adolescent children together to discuss and exchange views on topics such as gender, reproductive health and HIV/AIDS. A total of 160 intergenerational sessions were held, averaging 20 participants per session. At the end of this intervention, social mobilization activities brought the five target communities together.

Peer Educators were charged with selecting the discussion topics. An adult moderator was selected from the Technical Committee (comprised of representatives of institutions working on issues related to adolescent health and included, in addition to the Ministries, UNICEF, WHO, UNFPA, and Plan International). The peer educators served as facilitators.

This intervention activity provided a supportive environment for discussion through community advocacy, organization of educational activities, and outreach. Researchers surveyed both parents/guardians and youths to assess whether changes in communication patterns have started to emerge as a result of the intervention.

\section{Study limitations}

Despite taking every precaution, it is worth noting that there were limitations to this operational research study. It must be noted that the idea of a "control zone" that was used in this study did not fit well in the context of the actual reality. This is because in recent years a number of interventions focusing on Adolescent Reproductive Health have been implemented by the government and various NGOs there is really no locality in Senegal that is "virgin territory" in terms of RH interventions.

Information about RH is widely disseminated via the media, various other stakeholders, etc. Thus even if the selected control sites were not part of the intervention activities of this particular study, they would have been involved in other general RH activities as well as those specifically targeting youth.

To help facilitate the training of the Adult Peer Educators and the Youth Peer Educators a module on parent-child communication was developed with content based on the "Grandir en Harmonie" (Growing in Harmony) RH/HIV/AIDS Life Skills curriculum developed by the Ministry of Health and Population Council/FRONTIERS. Developing the curriculum for this module was not at all easy. The initial curriculum created by the consultant was too theoretical, 
too long, and in general too complex to be used by those who had little schooling. In the end it was decided to just use the Facilitator Worksheets.

During the intervention activities, the Peer Educators generally spent little time addressing some of the more technical RH topics such as reproductive anatomy, puberty and contraceptives. Instead discussions focused more on things such as social behavior, values, planned pregnancy, etc. Despite organizing one-day training for the facilitators to review these more technical RH topics, and to encourage more discussion about them, this problem remained.

To help overcome the reluctance of some of the parents/guardians and youth, a number of sensitization activities were conducted. Community forums and advocacy meetings were organized in all the intervention sites. These were open to everyone in the community, but they were particularly directed towards parents/guardians (male and female) heads of households. Unfortunately, despite myriad social mobilization and sensitizations activities, fewer men than women took part in the group discussions and Intergenerational Dialogue Sessions. In total, men accounted for only $32 \%$ of the parent/guardian participants.

The intervention activities for this study only lasted a few months due to the ending of the BRIDGE/CEDPA project. This short duration did not permit the interventions to reach the majority of parents/guardian and youths.

\section{Results}

The majority of the adolescents were girls (60\%), and the average age of the adolescents was 16 years. The majority of adolescents, during the time both surveys were conducted, were enrolled in school. The majority of the adult respondents were also female, with an average age of 43 years for the women and 54 years for the men. Their overall educational level was low and the majority had no formal schooling.

Several in-depth, qualitative, interviews demonstrated that kersa (a Wolof word that translates as: "modesty" as wells as "reserve, politeness or deference") is the main reason given by youths for explaining why they find it difficult to talk to their parents/guardians about reproductive health and sexual health issues, as the comments from the following young woman well illustrate: "I feel really uncomfortable discussing this subject with my parents. My father especially...I don't even dare imagine this type of conversation with him. And even with my mother, I always feel very embarrassed each time I talk to her about sex (...) In fact, you can just say that in Senegal it is never easy for a young person to sit down with his or her mother or father and talk about things like condoms or HIV/AIDS, etc. We just are not brought up to behave like that."

In addition to "kersa" some youths say their parents are not approachable and so they are not given a chance to discuss this issue. As one young man said: "Some parents automatically get defensive and do not have an open spirit for encouraging this kind of exchange, however when they are away from home, they are very open and communicative with other youths but at home, they are very firm and closed with their own children."

This feeling of a lack of openness on the part of parents/guardians is one of the reasons many young people give for being afraid to initiate discussions about RH with their parents saying they fear their parents will become suspicious about their reasons for doing so, especially if it's a girl. 
In fact, many girls said they prefer to avoid bringing up such subjects for this very reason. As one young girl said: "Sometimes I really do feel the need to discuss something with my father or my mother about certain RH questions that worry me...but my parents are not easy - especially my mother! If I pose such a question, she's going to automatically think that I'm pregnant or that I've started seeing boys...you have to understand that our parents don't always look at this with a "good eye" for them, posing questions about sexuality is taboo."

Responses from the adolescents indicate that their perceptions about the ease of discussing sexuality issues with their parents changed during the course of the project. In the endline survey, more adolescents said they found it easier to discuss reproductive health issues than in the baseline survey (38\% vs. 25\%). This improvement held true across gender, age, and residence; however, the difference was greatest for rural youth (40\% vs. $26 \%$ ).

The educational interventions conducted at the sites did contribute to improving communications between youth and adults on reproductive health issues and in increasing the level, quality, and frequency of communications between generations about RH and HIV/AIDS. However, at endline, there were important variations by age, gender, and location.

Overall, the proportion of adolescents who declared that they had discussed RH issues with their parents increased significantly between the baseline and endline surveys ( $57 \%$ to $62 \% \mathrm{p}=0.008$ ). At endline, a greater proportion of older adolescents (15-19 years), regardless of gender or where they lived, said they talked to their parents about RH matters than did those in the 10-14 year old age group (63\% vs. 59\%). Girls were much more likely than boys to talk about RH issues with their female parents ( $74 \%$ vs. $44 \%)$, but there was no difference reported for male parents.

This intervention contributed significantly to improving the quality of communication between youths and parents. At the time of the end-line survey, discussions were easier to initiate, more positive and more direct. At the endline survey, significantly more adolescents stated that they felt more comfortable discussing RH issues with their parents than at the baseline survey $-38 \%$ vs. $25 \%$, an improvement that was observed across gender, age and location categories. The proportion of youth who initiated talks about RH with their parents/guardians also increased and the increase was significant among youth in urban areas (22\% vs. $11 \% \mathrm{p}=0,000)$. At the baseline survey, a little more than half of the youths stated that they had the opportunity to ask questions during discussions about RH with their parents/guardians. At the endline survey, the proportion had increased significantly to $66 \%$. Moreover, at the baseline survey, $42 \%$ of the youth said they frequently talked with their parents/guardians about these issues, but at the endline survey this proportion had increased significantly to $51 \%$, and the proportion youth saying speaking "occasionally" or "infrequently" decreased to $14 \%$ from $40 \%$. The most significant improvement was in the 10-14 year old age group. Adult respondents also reported significant increases in discussing reproductive health-related subjects and in the frequency of communications.

Regardless of the age or place of residence, much more boys than girls stated they were sexually active. At the baseline survey, $29 \%$ of boys and $9 \%$ of girls reported being sexually active and this increased by the final survey to $33 \%$ of boys and $12 \%$ of girls. Only $25 \%$ of these sexually active adolescents reported using protection during the first intercourse, and this didn't change after the intervention. However, after the intervention, significantly more sexually active youth reported using protection during last sex (27\% vs. $46 \%)$. Condoms were the main method used by boys and girls. 
The attitudes of parents/guardians in communicating information on sexual issues to youth were strongly linked to gender. Discussions with girls center on abstinence and chastity to avoid pregnancy, whereas with boys, STI/HIV/AIDS issues are more openly discussed, implying that parents are more accepting of sexual activity by boys. Contraception remains a sensitive issue that was not discussed very much, even after the intervention.

\section{Lessons Learned}

This study demonstrates that, within the Senegalese cultural context, despite the sensitivity associated with discussing sexuality, it is possible to develop a program that can help improve the quantity and quality of communications between young people and adults. The intervention encouraged sharing of information among stakeholders, parents, guardians and youth, while also allowing for the promotion of traditional values such as abstinence before marriage and, if not abstinence, then responsible sexual behavior (contraception and condom use). The intervention served to create a time and space for Intergenerational Dialogue, and in doing so contributed to renewing the traditional ties between parents and adolescents in Senegalese families. Throughout the duration of the project, efforts to mobilize political, administrative, religious, and community leaders continued, which contributed greatly to the success of the intergenerational dialogues. Partnerships established with the ministries facilitated the implementation of the project. This model of intergenerational communication is now included within the interventions for adolescents implemented by the Senegalese Ministry of Youth, and CEDPA has replicated the model in South Africa. 


\section{REMERCIEMENTS}

L'équipe de recherche adresse ses vifs remerciements à toutes les personnes qui se sont investies dans la réalisation de ce travail. Leur engagement a contribué à son succès. Il s'agit de nos partenaires du CEDPA, particulièrement de Soukeye Dieng, Alimata Deme, Djibi Sow, Babacar Ndiaye et Jean Sagne

Du Ministère de la Santé à travers la Division de la Santé de la Reproduction (DSR), ses équipes cadres de région et de district à Kaolack et du Ministère de la Jeunesse au niveau Central et régional. Particulièrement au Dr Aboubacry Sy et à Mr Ciré Lo, respectivement chef du bureau adolescent à la DSR et Directeur de la Jeunesse.

L'exécution de cette étude a été facilitée par l'appui et la disponibilité d'institutions et personnes qui composent les comités de pilotage au niveau national et local.

Nos remerciements vont aussi à l'endroit des enquêteurs et enquêtrices qui ont contribué de façon significative à la réussite de ce travail, souvent dans des conditions difficiles. Leur détermination et leur savoir-faire ont été décisifs dans la qualité des informations recueillies. Nous associons à ces remerciements les agents de saisie dont la célérité nous a permis de déposer des fichiers de données dans un temps record.

Cette liste n'est certainement pas exhaustive, plusieurs personnes ayant apporté une précieuse contribution pour la réalisation de ce travail. Qu'elles trouvent ici l'expression de notre sincère reconnaissance! 


\section{Table des matières}

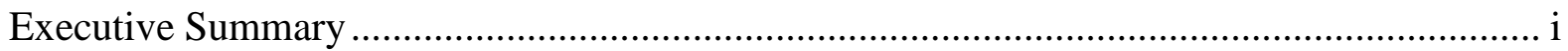

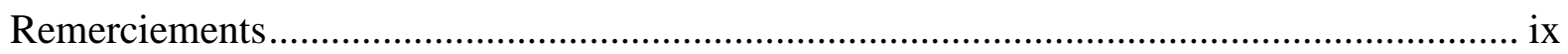

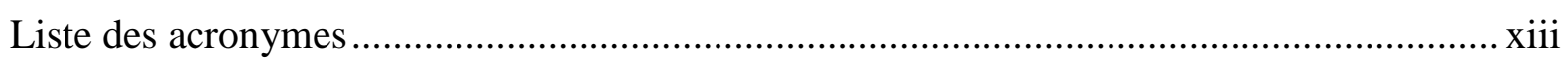

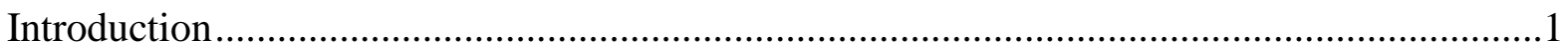

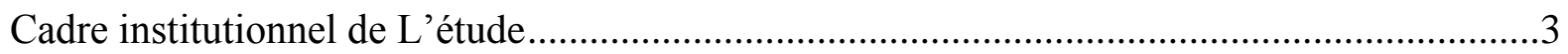

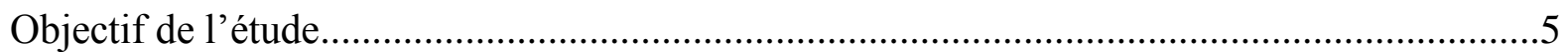

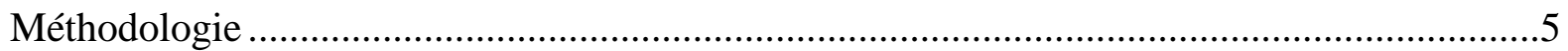

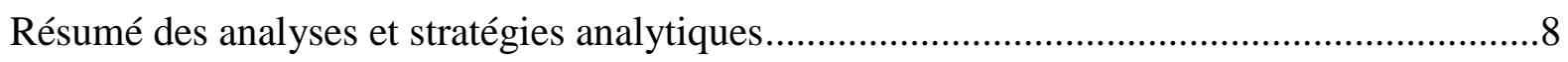

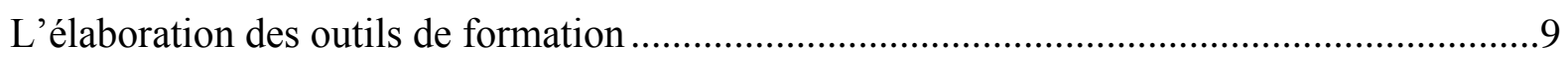

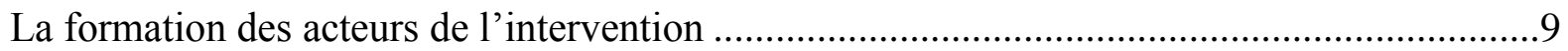

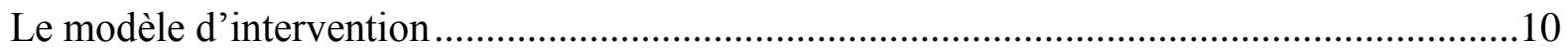

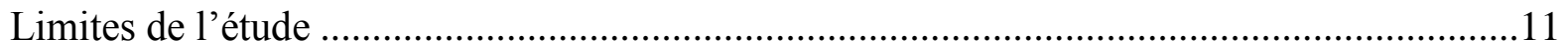

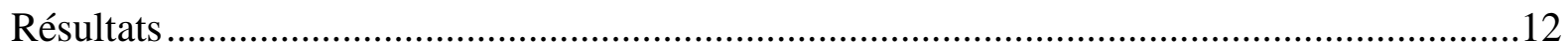

Communication entre parents/tuteurs en matière de SR/VIH/SIDA ........................................16

Le niveau de la communication entre parents et adolescent(e)s............................................19

La qualité de la communication entre adolescent(e)s et parents...............................................22

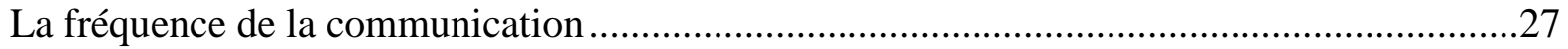

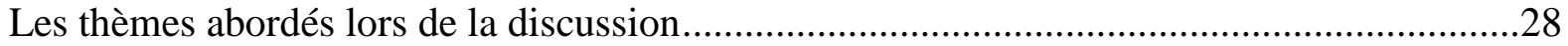

Attitude des adolescent(e)s vis-à-vis des programmes d'amélioration de la

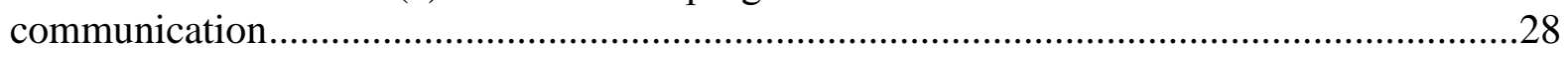

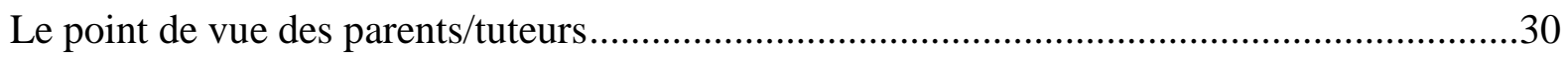

Préférence des parents en matière de discussion ....................................................................32

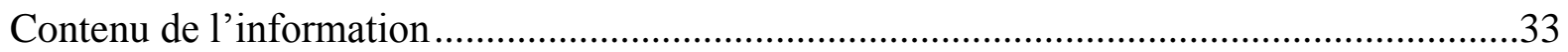

Compétence des adolescent(e)s en matière de SR ................................................................

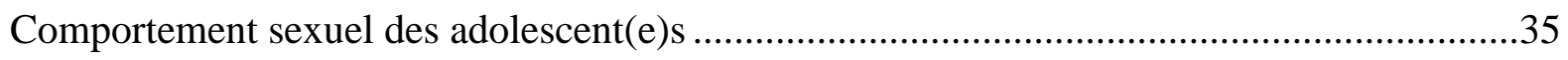

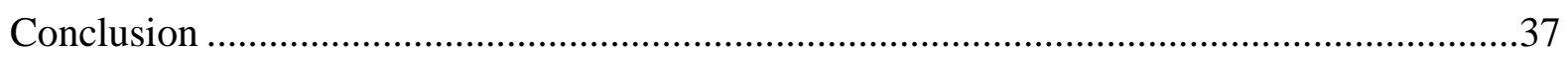

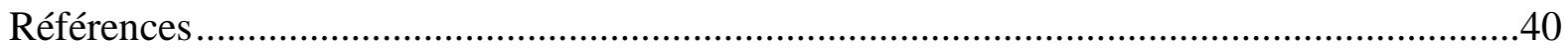




\section{Table des tableaux}

Tableau 1 : $\quad$ Bilan de collecte (Nombre absolu) ...........................................................

Tableau 2: Caractéristiques sociodémographiques des adolescent(e)s (en \%)................13

Tableau 3: Répartition des adolescent(e)s selon le sexe, le lieu de résidence et la cohabitation avec les parents $(\mathrm{en} \%)$.......................................................15

Tableau 4 : $\quad$ Répartition des parents selon certaines caractéristiques (en \%) ....................16

Tableau 5: $\quad$ Répartition des garçons et filles selon la réaction des parents à leurs questions lors des discussions (en \%) .....................................................26

Tableau 6: $\quad$ Répartition (\%) des adolescent(e)s selon les aspects de la SR abordés lors des discussions avec les parents selon le sexe

Tableau 7: $\quad$ Répartition (\%) des adolescent(e)s selon qu'ils aimeraient ou non que les parents discutent plus avec eux des questions liées à la SR selon l'âge et le sexe.

Tableau 8: $\quad$ Répartition (\%) des adolescents selon leur connaissance des risques liés aux rapports sexuels et des méthodes pour éviter ou retarder une grossesse

Tableau 9: $\quad$ Répartition (\%) des adolescents selon l'utilisation d'une méthode de protection lors du premier rapport sexuel et lors du dernier rapport sexuel 


\section{Table des Graphiques}

Schéma 1. Le cadre institutionnel de l'étude............................................................4

Graphique 1 : Répartition ( \%) des adolescent(e)s selon la perception par rapport à la facilité de discuter de la SR avec leurs parents, suivant le milieu de résidence

Graphique 2: Répartition ( \%) des adolescent(e)s selon la perception par rapport à la facilité de discuter de la SR avec leurs parents, suivant l'âge et le genre

Graphique 3 : Répartition ( \%) des adolescent(e)s qui déclarent discuter des questions de SR selon le milieu de résidence

Graphique 4 : Répartition ( \%) des adolescent(e)s qui déclarent discuter des questions de SR selon le genre et l'âge

Graphique 5 : Répartition des adolescents qui discutent de SR avec les parents, suivant la personne qui prend l'initiative de la discussion et le lieu de résidence

Graphique 6: Répartition des adolescents qui discutent de SR avec les parents, suivant la personne qui prend l'initiative de la discussion, le genre et l'âge

Graphique 7: Répartition des adolescent(e)s selon qu'ils aient ou non l'opportunité de poser des questions lors des discussions avec leurs parents/tuteurs (en $\%)$

Graphique 8: Répartition des adolescents selon la fréquence de la discussion avec les parents/tuteurs (en \%)

Graphique 9: Répartition (\%) des parents/tuteurs qui communiquent avec les adolescent(e)s selon le sexe

Graphique 10: Répartition (\%) des parents selon l'enfant avec lequel ils communiquent le plus, en fonction du sexe

Graphique 11: Répartition (\%) des adolescent(e)s selon qu'ils aient déjà eu ou non une expérience sexuelle, selon l'âge et le sexe 


\section{LISTE DES ACRONYMES}

APROFES Association pour la Promotion de la Femme Sénégalaise

BRIDGE Building Reproductive health through Improved Democracy, good Governance and Equity for women and youth

CEDPA Centre for Development and Population Activities

CEFFEVA Comité d'Etudes sur la Femme la Famille et l'Environnement en Afrique

EDS Enquêtes Démographiques et de Santé

ICP Infirmier(ère) Chef de Poste

IEC Information, Education, Communication

IST Infection Sexuellement Transmissible

OMS Organisation Mondiale de la Santé

ONUSIDA Organisation des Nations Unies pour la lutte contre le SIDA

RPSR Réseau pour la Promotion de la Santé de la Reproduction des jeunes de Kaolack

SDI Session de Dialogue Intergénérationnel

SIDA Syndrome de l'Immunodéficience Acquise

SDDC Service Départemental du Développement Communautaire

SR Santé de la Reproduction

SRA/J Santé de la Reproduction des Adolescent(e)s/Jeunes

UNFPA United Nation Fund for Population Activities

USAID United States Agency for International Development

VIH Virus de l'Immunodéficience Humaine

RPSR Réseau pour la Promotion de la Santé de la Reproduction 


\section{INTRODUCTION}

Traditionnellement, il y avait dans la société sénégalaise un fort système de régulation des adolescent(e)s, fondé sur les enseignements religieux, les coutumes et l'éducation transmise par la famille étendue. Une forme d'éducation sexuelle était aussi transmise par la famille étendue. Cette éducation avait pour but de préparer les jeunes adultes à assumer pleinement les statuts et rôles qui leur sont destinés dans la société. Il s'agit principalement des rôles d'époux ou d'épouses, de mères et de pères de familles.

Si pendant longtemps ce modèle d'éducation a fonctionné en permettant aux plus jeunes membres de la communauté d'entrer pleinement dans la vie adulte et de prendre très tôt conscience des risques liés à la sexualité précoce, il n'en demeure pas moins que, dans le contexte actuel, ces pratiques ont de plus en plus tendance à s'effriter et laisse place à un nouveau modèle dont la principale caractéristique est le relâchement des modes traditionnelles de socialisation. En effet, dans le contexte actuel, les familles encadrent de moins en moins les jeunes. Ceux-ci sont très tôt livrer à eux-mêmes et entrent dans la vie sexuelle sans y être convenablement préparé. Cette situation résulte de la transformation des modes traditionnelles d'encadrement des jeunes du fait, notamment, de la modernisation et de la globalisation de la culture à travers les médias, l'internet, le cinéma et la vidéo. La conséquence immédiate de cette situation, est la transformation des mécanismes traditionnels de régulation par la famille étendue et des relations parent/enfant. Cette situation contribue aussi à creuser l'écart entre les parents/adultes et leurs enfants et à limiter les possibilités de dialogue intergénérationnelle.

$\mathrm{Du}$ fait de cette situation, il semble nécessaire pour augmenter les connaissances des adolescent(e)s en matière de SR/VIH/SIDA et promouvoir des attitudes plus responsables, de renforcer la communication entre les parents et les adolescent(e)s sur les questions de SR. Toutefois, comme le montrent certaines recherches déjà menées sur cette problématique (Jaccard et al., 1996; Luster et Small, 1997; Miller et al., 1999), le renforcement de la communication entre les parents et les enfants sur les questions de SR, à lui seul, ne suffit toujours pas pour protéger les adolescent(e)s des comportements sexuels à risques. Leur "connexion" avec leurs parents et aussi d'autres modes de régulations sociales et familiales peuvent, en outre, être des facteurs protecteurs. Le renforcement de cette connexion requiert, toutefois, l'établissement d'une relation favorable, par laquelle une information correcte et positive pourra être transmise par les parents à leurs enfants.

Si aux USA, la littérature sur communication entre les parents et les adolescents est abondante, dans le contexte africain, par contre, les études portant sur la question sont rares. En effet, les quelques travaux qui existent sont essentiellement des études diagnostiques qui montrent l'importance d'une telle communication dans la vie des jeunes et la nécessité pour les parents d'établir le dialogue avec leurs enfants. Plusieurs études montrent que l'essentiel des informations dont disposent les jeunes, en Afrique, sur la sexualité et la santé de la reproduction provient de leurs amis et des médias (Pacific Institute for Women's Health 2002; Dieng et al. 2001). La passerelle entre la communication parent-enfant et la comportement sexuel est en début d'exploration. Une étude effectuée au Sénégal (CEFFEVA, 2001) a montré que les adolescents et leurs parents ont une perception différente de la communication sur la sexualité. En effet, selon les résultats de cette étude, les adolescents considèrent que les discours se concentrent d'avantage sur les conséquences des rapports sexuels avant le mariage et ne donnent 
pas suffisamment d'informations précises sur les questions liées à la prévention des grossesses et des MST. Les parents quant à eux considèrent qu'en donnant à leurs enfants des informations ayant trait à la sexualité, ils encouragent ces derniers à avoir des rapports sexuels. En plus, ils hésitent de parler à leurs enfants de la prévention parce qu'ils croient qu'ils n'ont pas eux même les bonnes informations (Dieng et al. 2001). En général, la recherche a montré qu'au Sénégal, la communication entre les parents et les adolescents en matière de sexualité est quasi inexistante, et même lorsqu'elle a lieu, elle est à sens unique et ne répond pas toujours aux préoccupations des jeunes. Les parents parlent mais n'écoutent pas les préoccupations des jeunes.

Malgré les difficultés qu'ils rencontrent pour aborder les questions de sexualité avec leurs enfants, les parents sénégalais, se rendent compte de l'importance d'avoir de telles discussions avec leurs enfants et des besoins de ces derniers pour avoir de plus amples informations sur ces questions. Du fait de cette situation, les parents veulent s'impliquer d'avantage dans l'éducation sexuelle des enfants et renforcer cette communication (Dieng et al., 2001; Diop et al., 2004). En plus, les jeunes disent qu'ils préféraient obtenir les informations de leurs parents au lieu de l'obtenir de leurs pairs ou des autres. (Dieng et al. 2001). Ainsi, après une série d'interventions pour améliorer le santé de la reproduction, les adolescents ont cherché plus d'information auprès des adultes comparé à leurs amis, mais ils n'ont pas rapporté une amélioration de la communication parent-adolescent.(Diop et al. 2004).

Dans ce cadre, pour contribuer à l'amélioration de la communication entre les parents et les adolescent(e)s et promouvoir le dialogue intergénérationnel sur les questions de SR/VIH/SIDA, le Population Council a mis en place, en collaboration avec le Centre pour le développement des activités de population (CEDPA), le Ministère de la Santé et le Ministère de la Jeunesse une recherche opérationnelle destinée à avoir une meilleure compréhension des interventions conçues spécifiquement pour améliorer la communication entre les parents/tuteurs et les adolescent(e)s en matière de santé de la reproduction/VIH/SIDA. Les résultats de cette étude devraient servir de base à l'élaboration de futures stratégies et programmes pour la promotion de la SR des adolescent(e)s et prendre en charge les besoins d'informations des adolescent(e)s. Pour ce faire, l'étude a eu lieu dans le département de Kaolack et à l'intérieur de celui-ci quatre communautés rurales et un quartier de la ville ont été choisis pour abriter les diverses activités qui ont été menées dans le cadre de cette recherche (les activités de recherche et les interventions dans la communauté). Un quartier de la ville et une communauté rurale ont aussi été choisis comme de zone témoin. Ces deux derniers sites n'étaient pas concernés par les activités d'interventions.

Les activités d'intervention qui ont été menées dans la communauté avaient pour but de promouvoir chez les adolescent(e)s des comportements et des attitudes protecteurs par le biais d'une communication accrue entre les parents et les adolescent(e)s en matière de SR/VH/SIDA. Pour ce faire, des pairs éducateurs issus des différentes communautés où l'étude a lieu ont été recrutés. Ces derniers ont été ensuite formés pour animer 380 séances de causerie éducatives pour 1600 parents, 380 séances de compétence pour la vie pour 1600 adolescent(e)s(e)s et 228 sessions de dialogue intergénérationnel pour tout le monde. Ces animateurs, membres de leur communauté, ont été appuyés dans leur mission par un comité local de pilotage qui regroupe en son sein des autorités, leaders d'opinion et personnes ressources de la localité. La mission de ce comité étant essentiellement tournée vers le plaidoyer et la mobilisation sociale.

Pour une pleine participation des adolescent(e)s et de leurs parents (ou tuteurs), des fora communautaires, des émissions de radio et des activités théâtrales aussi ont été organisées dans 
les différents sites de l'étude. Le processus de mise en œuvre des interventions a duré 10 mois (juillet 2005-avril 2006) et a vu la participation de 1512 parents et 1528 adolescent(e)s. Le niveau de participation aux différentes activités est très satisfaisant : $96 \%$ de parents ont pu assister à au moins trois séances de causerie éducative et $72 \%$ ont pu suivre les cinq séances prévues pour chaque groupe. Chez le groupe d'adolescent(e)s, ces taux sont respectivement de l'ordre de $99 \%$ et $85 \%$. En ce qui concerne la participation aux SDI, 92\% d'adultes et 93\% d'adolescent(e)s environ ont participé aux deux SDI prévues pour chaque participant.

\section{CADRE INSTITUTIONNEL DE L'ÉTUDE}

Cette recherche opérationnelle porte sur l'amélioration de la communication entre parents (ou tuteurs) et enfants sur les questions de SR/VIH/SIDA. Concrètement, il s'agissait de tester l'efficacité d'un modèle d'intervention spécifiquement conçu pour améliorer le niveau et la qualité de la communication entre les parents/tuteurs et les adolescent(e)s sur les questions de SR. Pour ce faire, une approche multi-sectorielle a été adoptée pour permettre l'implication de plusieurs acteurs intervenant dans le domaine de la santé de la reproduction: le Centre pour le développement des activités de population (CEDPA) a assuré la mise en œuvre des interventions et le Population Council a mené les enquêtes de base et d'évaluation ainsi que la documentation de tout le processus. Ces deux institutions ont travaillé en étroite collaboration avec le ministère de la santé et de la prévention médicale (à travers notamment la Division de la Santé de la Reproduction et la région médicale de Kaolack) et celui de la jeunesse (Direction de la Jeunesse et de la Vie Associative). Cette collaboration fut ensuite élargie à d'autres institutions travaillant dans le domaine de la santé de la reproduction (USAID, OMS, UNFPA, UNICEF, PPJ, ADEMAS, GEEP etc.). Des réunions ont été périodiquement organisées afin de s'assurer qu'il y avait une compréhension commune du projet entre les différentes parties prenantes. Ce processus de discussion et de négociation a mis en exergue les aspects critiques de l'étude et a favorisé son appropriation par chacun des acteurs qui était impliqué dans le projet. Les comités ci-dessous ont été constitués pour suivre l'évolution du projet.

\section{Le comité scientifique :}

Ce comité a été crée par les deux organisations partenaires de l'étude (Population Council et CEDPA). En plus de ces deux institutions le comité scientifique regroupe les autres partenaires que sont les ministères de la santé et de la prévention médicale et celui de la jeunesse, 1'USAID, l'OMS, le FNUAP, l'UNICEF et Projet de promotion des jeunes (PPJ) du ministère de la jeunesse. Ce comité avait pour rôle d'apporter un appui technique, administratif et organisationnel à l'exécution de l'étude. Il a ainsi a guidé l'élaboration du protocole de recherche, le choix des sites de l'étude, la conception des questionnaires et des stratégies d'interventions (le plan opérationnel de l'intervention et le tableau synoptique des activités des acteurs de l'intervention, enfin les outils de suivi des activités des relais)

\section{Le comité départemental :}

Ce comité regroupe en son sein l'équipe cadre du district, certains responsables des services techniques du département, l'inspecteur départemental de la jeunesse et le responsable du Service Départemental du Développement Communautaire (SDDC). Le RPSR et certaines 
associations intervenant directement sur les cibles jeunes et femmes/famille étaient également représentés. Enfin, par souci de créer une passerelle entre le niveau départemental et le niveau opérationnel, les communautés dans lesquelles se déroulaient les interventions étaient également représentées par un de leurs membres. Le CTDP est l'organe d'encadrement des activités de l'étude. Son action a aidé à l'acceptation du projet par les autorités et les communautés, ainsi que leur pleine participation aux différentes activités. Elle a beaucoup aidé dans la coordination et le suivi des activités de l'étude.

\section{Le niveau local :}

En tant qu'organe d'exécution des activités de l'intervention, le comité local de pilotage a réuni en son sein l'équipe d'animation des activités de l'intervention, notamment l'infirmier chef de poste, les relais parents, les pairs éducateurs et les modérateurs. Il est élargi aux différentes entités de la localité qui seraient à mesure d'apporter une aide quelconque dans la mobilisation communautaire ou dans la recherche de solutions pratiques à certains problèmes que rencontrerait éventuellement l'équipe d'animation.

\section{Schéma 1. Le cadre institutionnel de l'étude}

COMITE SCIENTIFIQUE

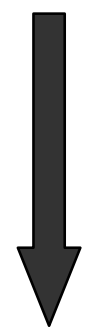

COMITE DE PILOTAGE AU NIVEAU DEPARTEMENTAL

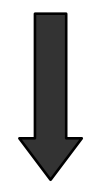

COMITE DE PILOTAGE AU NIVEAU LOCAL
DSR (Direction de santé de la reproduction du Ministère de la santé)

DJVA (Direction de la jeunesse et de la vie associative du Ministère de la jeunesse)

PPJ (Projet de Promotion des jeunes du Ministère de la jeunesse)

Population Council /FRONTIERS

$\Rightarrow$ CEDPA /BRIDGE

$\Rightarrow$ USAID

$\Rightarrow$ OMS

$\Rightarrow$ FNUAP

$\Rightarrow$ UNICEF

$\Rightarrow \quad$ Médecin chef du district médical de Kaolack

$\Rightarrow \quad$ Inspecteur départemental de la jeunesse

Chef du service départemental du développement communautaire

$\Rightarrow \quad$ Réseau pour la proportion de la SR

$\Rightarrow \quad$ ONG ayant pour cibles femmes/jeunes

$\Rightarrow \quad$ Membres d'associations

$\Rightarrow \quad$ ICP (Infirmier chef de poste)

Ensemble des relais parents/adultes

Ensembles des relais jeunes

Ensemble des modérateurs

$\Rightarrow$ Associations villageoises etc. 


\section{OBJECTIF DE L'ÉTUDE}

L'objectif de cette étude est d'obtenir une meilleure compréhension des interventions conçues spécifiquement pour améliorer la communication entre les parents et les adolescent(e)s en matière de santé de la reproduction. Plus spécifiquement, il s'agit dans cette recherche d'évaluer la fonctionnalité d'un modèle d'intervention destiné à prendre en charge la communication adulte/adolescent(e)s en termes de changements sur:

- Les connaissances et attitudes des parents et des jeunes sur des questions concernant les adolescent(e)s, notamment la santé la reproduction.

- L'appui communautaire pour les besoins des adolescent(e)s, en particulier dans la communication sur les questions de santé de la reproduction des adolescent(e)s.

- La fréquence, qualité et les sujets discutés pendant la communication parent - enfant.

- Les mécanismes de protection et de régulation dont se servent les parents pour prendre en charge la santé de la reproduction des adolescent(e)s.

Le projet contribuera au résultat intermédiaire (IR2) de FRONTIERS: «Connaissances générées, organisées et communiquées pour faire avancer les meilleures pratiques » et répondra à l'engagement du CEDPA de prendre en charge la communication parent - enfant dans le cadre de son mandat à l'OS3 de l'USAID "accroître l'utilisation des services de SR."

\section{MÉTHODOLOGIE}

\section{Le choix des sites de l'étude}

L'étude est circonscrite dans la région de Kaolack et plus particulièrement dans le département du même nom ${ }^{1}$ où s'est déroulé l'essentiel des activités qui ont été menées dans le cadre de cette étude (recherche, intervention). Le choix du département de kaolack comme site de l'étude se justifie par le fait qu'il est une zone d'intervention pour le RPSR (Réseau de Promotion de la Santé de la Reproduction), un partenaire du programme bilatéral USAID, particulièrement actif dans la promotion de la santé de la reproduction des adolescent(e)s dans la région de kaolack. En effet, ce réseau qui est essentiellement composé de jeunes issus des différentes localités mène de nombreuses activités en vue de la promotion et de l'amélioration de la santé de la reproduction dans le département.

Outre, la possibilité de s'appuyer sur un réseau de jeunes travaillant depuis de nombreuses années dans le domaine de la SR, il faut aussi signaler que Kaolack est une ville carrefour à la frontière de la Gambie et en raison de sa position de centre de commerce de l'arachide, elle attire les populations des zones périphériques et par conséquent possède une importante population de travailleuses du sexe. Pour preuve, si au plan national, le taux de prévalence du VIH est relativement faible au Sénégal ${ }^{2}$ (EDS 2005), dans la région de Kaolack, la situation devient plus

\footnotetext{
${ }^{1}$ Dans le centre du pays, a $192 \mathrm{~km}$ de Dakar la capitale

${ }^{2}$ Ce taux étant estimé à environ 1,4\% pour l'ensemble du pays (EDS, 2005)
} 
critique avec un taux de prévalence régional de l'ordre de 1,8\% dans la population générale et un taux de 37\% parmi les travailleuses du sexe (EDS, 2005).

Au total, quatre (4) communautés rurales et un (1) quartier de la ville ont été finalement choisies parmi les 13 localités où intervient le RPSR comme zone d'intervention et de recherche. Un quartier de la ville et une communauté rurale ont été aussi choisis comme zone témoin. Ces deux dernières localités n'ont été concernées que par le volet recherche du programme. Aucune activité d'intervention n'y ont été menées. Le choix des différents sites de l'étude a été fait à l'issue d'un diagnostic préliminaire mené par le Population Council en collaboration avec le RPSR. Les interventions dans les zones rurales sont justifiées par la rareté des interventions en faveur de la santé de la reproduction parmi les jeunes populations rurales.

\section{Les procédés de recherche}

La recherche a comporté trois phases : une phase pré-test ou enquête de base dont l'objectif était de mesurer la situation de départ, une phase d'intervention menée par CEDPA dans les différents sites qui ont été choisis dans l'étude, une phase post-test ou enquête d'évaluation finale. L'étude utilise un motif de contrôle avec une aire de comparaison. Ces unités d'analyse sont les adolescent(e)s et les adultes dans la communauté qui participent à l'intervention.

\section{$\begin{array}{llll}\text { Groupe expérimental } & O_{1} & X & O_{2}\end{array}$ \\ $\begin{array}{lll}\text { Groupe de comparaison } & \mathrm{O}_{3} & \mathrm{O}_{4}\end{array}$}

Où:

$\mathbf{O}_{1}$ et $\mathbf{O}_{3}=\quad$ Enquête pré test pour mesurer la connaissance des adultes et des jeunes, les attitudes et pratiques concernant la santé de la reproduction et la communication intergénérationnelle. L'enquête pré-test sera menée après le plaidoyer des activités dans la zone expérimentale et dans la zone de comparaison.

$\mathbf{X}=$ Activités d'intervention

$\mathbf{O}_{2}$ et $\mathbf{O}_{4}=$ Enquête post-test pour mesurer la connaissance des adultes et des jeunes, les attitudes et pratiques concernant la santé de la reproduction et la communication intergénérationnelle. L'enquête post-test sera menée 10 mois après que l'intervention ait eu lieu dans la zone où les interventions ont été menées (zone expérimentale).

\section{La collecte des données}

Cette recherche opérationnelle a comporté plusieurs étapes. Dans un premier temps une enquête de base a été effectuée avant les interventions. Le but de cette première phase de l'étude était de recueillir des informations suffisamment détaillées pour rendre compte avant les interventions du niveau, de la fréquence et de la qualité de la communication entre parents et enfants en matière de santé de la reproduction/VIH/SIDA. Un questionnaire a été administré spécifiquement aux adolescent(e)s et un autre à leurs parents/tuteurs. Chacun des thèmes abordés dans les questionnaires devait renseigner sur un aspect particulier de la problématique étudiée. 
A l'issue de l'enquête de base des activités d'intervention ont été ensuite menées. Ces activités avaient pour but de renforcer les compétences des bénéficiaires sur ces questions et d'améliorer le niveau et la qualité de la communication entre les parents/tuteurs et les adolescent(e)s en matière de santé de la reproduction. Elles ont consisté pour l'essentiel à des plaidoyers, des séances de sensibilisation et des formations en direction des adolescent(e)s et de leurs parents. Ces activités ont duré quatre mois (septembre 2005 - décembre 2006). Elles ont été immédiatement suivies d'investigations plus poussées de type qualitatif. Dans ce cadre, il s'agissait à travers une approche socio-anthropologique de comprendre et d'expliquer comment dans les familles sénégalaises les besoins d'informations des adolescent(e)s en matière de SR sont pris en charge. Cette enquête a également permis d'identifier les mécanismes de la communication entre parents et enfants en matière de SR et l'environnement dans lequel cette communication s'effectue. Ces données qualitatives ont été recueillies à travers les focus group et les récits de vie.

La phase post-test ou enquête d'évaluation quantitative est la dernière étape de ce long processus. Elle a eu lieu en décembre 2006, soit 12 mois après la fin des interventions. Dans cette dernière phase de l'étude, il s'agissait d'identifier les changements qui ont eu lieu dans la communauté en ce qui concerne la communication entre parents et enfants sur les questions de SR. Plus spécifiquement, il s'agissait de voir comment les interventions qui ont été menées dans la communauté ont contribué à l'émergence de nouvelles pratiques et de nouveaux comportements en matière de communication intergénérationnelle ?

Lors des différentes opérations de collecte des données, l'équipe s'est attachée à respecter les principes d'éthique qu'implique toute recherche. Les personnes interrogées ont été informées des objectifs de l'étude, du caractère confidentiel et anonyme du questionnaire. L'entretien n'était poursuivi que si la personne était librement consentante.

\section{Bilan de la collecte des données}

Au total 1293 adolescent(e)s et 896 parents/tuteurs ont été interrogés à l'enquête de base, alors que pour l'évaluation 1160 adolescent(e)s et 762 parents ont été interviewés (Tableau 1).

Tableau 1 : Bilan de collecte (Nombre absolu)

\begin{tabular}{|c|c|c|c|c|c|c|}
\hline & \multicolumn{2}{|c|}{ Urbain } & \multicolumn{2}{|c|}{ Rural } & \multicolumn{2}{|c|}{ Total } \\
\hline & $\begin{array}{l}\text { Enquête } \\
\text { de base }\end{array}$ & $\begin{array}{c}\text { Enquête } \\
\text { finale }\end{array}$ & $\begin{array}{l}\text { Enquête } \\
\text { de base }\end{array}$ & $\begin{array}{c}\text { Enquête } \\
\text { finale }\end{array}$ & $\begin{array}{l}\text { Enquête } \\
\text { de base }\end{array}$ & $\begin{array}{c}\text { Enquête } \\
\text { finale }\end{array}$ \\
\hline \multicolumn{7}{|l|}{ Garçons } \\
\hline $10-14$ ans & 29 & 22 & 98 & 38 & 127 & 60 \\
\hline $15-19$ ans & 115 & 75 & 279 & 116 & 394 & 191 \\
\hline \multicolumn{7}{|l|}{ Filles } \\
\hline $10-14$ ans & 47 & 150 & 142 & 205 & 189 & 355 \\
\hline 15-19 ans & 182 & 224 & 401 & 330 & 583 & 554 \\
\hline \multicolumn{7}{|l|}{ Total } \\
\hline Adolescents & 373 & 471 & 920 & 689 & 1293 & 1160 \\
\hline Parents & 262 & 172 & 644 & 590 & 906 & 762 \\
\hline
\end{tabular}


On remarque qu'il y a eu une légère baisse des taux de réponses dans l'enquête finale et ceci aussi bien pour les adolescent(e)s et les parents/tuteurs. Du côté des adolescent(e)s, on peut penser que la période durant laquelle l'enquête finale a été menée explique beaucoup la baisse des taux de réponses constatée dans cette dernière phase de l'étude. En effet, alors que l'enquête de base s'est fait pendant les vacances scolaires l'enquête finale a été menée durant l'année scolaire. Les adolescent(e)s étant pratiquement tous élèves, les enquêteurs étant le plus souvent dépendant des horaires des adolescent(e)s. En outre, il faut également signaler pour des raisons scolaires certains élèves avaient déménagé.

\section{RÉSUMÉ DES ANALYSES ET STRATÉGIES ANALYTIQUES}

Pour préparer l'analyse des données, les opérations suivantes ont été réalisées :

- Sortie des fréquences simples de toutes les variables des différents questionnaires pour inspection et correction au besoin

- Comparaison des échantillons obtenus avant et après interventions en vue de vérifier leur caractère superposable

L'analyse des données proprement dite a suivi le schéma ci-après :

1) Analyse bivariée des caractéristiques personnelles des adolescent(e)s et de leurs parents selon la zone de résidence (urbain-rural), le moment de l'enquête (enquête de base et évaluation) et l'âge. Ces analyses ont permis de voir si les caractéristiques des participants à l'étude qui ont été choisies, particulièrement celles qui sont associées aux principaux résultats, étaient à peu pré-identiques selon la zone de résidence, en considérant globalement les échantillons d'enquêtés (enquête de base et enquête finale).

2) Analyse bivariée de comparaison des indicateurs avant et après les interventions pour chaque zone de résidence. Cette analyse a permis de mesurer les évolutions qui ont eu lieu dans les différents sites de l'étude en ce qui concerne la communication entre les parents et les enfants sur les questions de SR.

L'analyse bivariée a utilisé des tests de comparaison de moyennes et de proportions. Ces tests ont été essentiellement mis en œuvre sous le logiciel STATA 8.

\section{Description des interventions}

La mise en œuvre des interventions s'est déroulée en trois étapes avec une longue période de préparation (phase d'élaboration des outils de l'intervention), une période de formation des acteurs de l'intervention, et une période au cours de laquelle le modèle est mis en œuvre dans les communautés. 


\section{L'ÉLABORATION DES OUTILS DE FORMATION}

\section{Le module de communication parents/enfants}

Afin de faciliter la formation des relais et pairs éducateurs, un module en communication parents/enfants a été développé. Pour sa conception, les curricula sur les compétences pour la vie en matière de SR/VIH/SIDA développés par CEDPA et Grandir en Harmonie élaboré par le Ministère de la Santé et FRONTIERS ont servi de base de travail. Ce curriculum était destiné aux acteurs de l'intervention (pairs éducateurs, relais parents, superviseurs et modérateurs). Il s'articule autour de trois thèmes majeurs: les valeurs, les questions liées à la situation d'adolescence et à la Santé de la Reproduction de l'adolescent(e)s(e) et les aspects liés à la communication. Dans le module il s'agissait de renforcer les connaissances des parents sur les difficultés que rencontrent les adolescent(e)s en matière de SR, sur les attentes des parents vis-àvis de leurs enfants et ceux des enfants vis-à-vis de leurs parents, sur le rôle des parents dans la détermination du chemin de vie de leurs enfants.

\section{Les fiches d'animation des relais et pairs éducateurs}

Des fiches d'animation de causeries et de session de dialogue intergénérationnel ont été conçues à partir du curriculum pour servir de guide d'animation pour les relais parents et adolescent(e)s. Cinq thèmes étaient retenus pour les séances de causeries éducatives avec les parents (apprenons à communiquer avec notre enfant ; perceptions et attentes des parents vis-à-vis de leur enfant; aidons nos enfants à prévenir la grossesse ; agissons pour la prévention des IST/VIH/SIDA chez les adolescent(e)s ; accompagnons les choix de nos enfants). Pour les adolescent(e)s ciblés, les causeries devaient installer des compétences de vie pour leur permettre de mieux se connaître, de renforcer leur estime et confiance en soi et d'être capables de prendre les décisions appropriées face aux multiples choix et défis de la vie. Afin de leur donner les moyens de communiquer avec leurs parents en découvrant leurs perceptions et les attentes de ces derniers, cinq fiches d'animation ont servi de support aux pairs éducateurs chargés de l'animation des séances de life skills avec les groupes d'adolescent(e)s.

\section{LA FORMATION DES ACTEURS DE L'INTERVENTION}

\section{L'identification et le recrutement des acteurs}

Les différents acteurs de l'intervention sont : les relais adultes ou parents, les éducateurs pairs jeunes, les superviseurs et les modérateurs des sessions de dialogue intergénérationnel. Pour la mise en œuvre des programmes, en partenariat avec CEDPA, le RPSR a formé 90 adultes en Santé de la Reproduction des Adolescent(e)s puis 60 pairs éducateurs jeunes en animation de sessions de compétences pour la vie. Ces derniers sont opérationnels dans les treize cellules du réseau. La quasi-totalité des vingt (20) relais adultes et vingt (20) pairs éducateurs retenus pour l'animation des différentes activités d'IEC vient de ce groupe.

La supervision des activités de ces relais a été confiée au poste de santé. Au total, dix superviseurs ont été retenus dont six (6) ICP. 
S'agissant du modérateur des séances de dialogue intergénérationnelle, il a été choisi au sein du comité de pilotage, sur proposition de ses membres et selon les mêmes critères d'éligibilité que les autres acteurs. La même personne n'étant pas forcément appelée à modérer deux sessions de suite, le nombre de modérateurs n'a pas été préalablement défini.

\section{Les ateliers de formation et de recyclage}

Trois ateliers de formation des acteurs, un atelier d'évaluation et un autre de recyclage des relais et d'orientation des modérateurs ont jalonné le processus de l'intervention. Un atelier de six jours a d'abord été organisé à l'intention des relais parents sélectionnés pour l'animation des séances de causerie éducative avec les adultes. Cet atelier a été suivi d'un autre regroupant essentiellement les pairs éducateurs qui ont été choisis pour animer les causeries destinées aux adolescent(e)s. Enfin, un troisième atelier d'une journée a été organiser pour l'orientation des autres acteurs de l'intervention (superviseurs et modérateurs).

Cet atelier a permis d'orienter les dix superviseurs retenus sur le module communication parents/enfants et de partager les outils de suivi des activités des relais. Cet atelier a également vu la participation des 19 relais parents et 19 pairs éducateurs déjà formés. Ce qui a permis, en plus, de préciser les tâches de chaque acteur et de répartir ces derniers au sein d'équipes d'animation. Une équipe est constituée d'un superviseur, de deux binômes de relais (adulte et jeune) et du modérateur qui l'assistent dans l'animation des sessions de dialogue intergénérationnel. Le superviseur en est le répondant.

\section{LE MODÈLE D'INTERVENTION}

Afin d'impulser la communication entre les parents et les adolescent(e)s au sein de la famille des sessions de dialogue intergénérationnel (SDI) ont été organisées dans les cinq sites de l'intervention. Il s'agissait à travers ces sessions de mettre ensemble, un groupe de 20 adolescent(e)s et adolescent(e)ses et un autre de 20 adultes, en vue de susciter un dialogue sur les questions liées à la SRA. Au cours de la session, chacun des participants doit nécessairement donner son avis, son point de vue sur une situation donnée tout en respectant celui des autres participants. La session étant animée par le binôme de relais (pair-éducateur, relais parent) avec la facilitation du modérateur, chacun des deux relais devait identifier au cours de ses séances de causerie les questions que les membres de son groupe aimeraient discuter avec l'autre génération. Le respect des engagements pris par les participants à communiquer une fois de retour dans leur famille est un autre mécanisme pour encourager la communication.

Les séances de causerie éducatives avaient pour but de faciliter le dialogue intergénérationnel en dotant d'une part les parents/adultes de connaissances en SRA, les adolescent(e)s en compétences pour la vie, d'autre part en technique de communication. Le binôme de relais devait progressivement et simultanément animer ses 5 séances pour permettre l'organisation des différentes SDI. Chaque animateur devait préparer sa séance sous le contrôle de son superviseur. Les performances des animateurs faisaient également l'objet d'un contrôle périodique de la part du superviseur au cours de la première ou de la deuxième séance de causerie, de la quatrième ou cinquième séance et enfin pendant les trois SDI. 
Des activités de mobilisation sociale ont été menées tout le long du processus. L'objectif visé à travers ces réunions était de vaincre les réticences des parents ou tuteurs d'adolescent(e)s encore sous l'emprise de certaines pesanteurs sociales et d'obtenir une pleine adhésion des familles. Pour ce faire, des informations claires et précises ont été apportées sur l'intervention, ses objectifs, les stratégies de communication et sa finalité. En plus de ces activités (foras communautaires et des réunions de plaidoyers), des émissions de radio et des activités théâtrales ont aussi été menées afin de renforcer cette communication de masse et de permettre une plus large diffusion du modèle de communication parents/adolescent(e)s. Ces émissions étaient diffusées dans deux des chaînes de radios les plus écoutées dans en zone rurale. Elles avaient pour objectif de poser au sein des familles le problème de la sexualité des adolescent(e)s et de ses implications, puis d'apporter la bonne information afin de susciter un intérêt à en discuter. Ainsi, en reprenant les différents thèmes enseignés par les relais, tout en posant la problématique actuelle de l'éducation sexuelle des jeunes, ces activités de communication de masse visaient à diffuser le modèle à l'échelle communautaire et à encourager le soutien et la pratique continue du dialogue intergénérationnel afin qu'il devienne désormais un réflexe communautaire.

\title{
LIMITES DE L'ÉTUDE
}

Bien que de nombreuses précautions ont été prises, cette recherche opérationnelle connaît cependant un certain nombre de limites qu'il convient de souligner.

\begin{abstract}
Absence de sites vierges
Quatre communautés rurales et un quartier de la ville de Kaolack ont été choisis. Deux autres sites ont aussi été choisis à l'intérieur du département de Kaolack (un quartier de la ville et une communauté rurale) pour servir de zone témoin (ou zone de contrôle). Dans ces deux derniers sites aucune activités d'intervention ne devaient être mené. Ces deux sites n'étaient donc concernés que par les activités de recherche.

La notion de zone témoin (ou de contrôle) auquel nous avons eu recours dans cette étude, n'est toutefois pas évidente dans le contexte actuel. En effet, du fait des nombreuses actions mis en œuvre au cours de ces dernières années par les pouvoirs publics et les ONG pour la promotion de la santé de la reproduction des adolescent(e)s aucune localité, au Sénégal, n'est actuellement vierge en matière d'intervention en santé de la reproduction. Ainsi, dans les deux sites qui ont servi de zone témoin, les informations sur la SR des adolescent(e)s sont véhiculées par les médias et les autres acteurs. En d'autres termes, même si ces deux sites n'ont pas abrité d'interventions tel que prévu par la recherche, il n'en demeure pas moins q'ils ont aussi bénéficié d'autres actions de SR en général et de SR des adolescent(e)s en particulier.
\end{abstract}

\section{Les outils de formation et de communication}

Afin de faciliter la formation et l'animation par acteurs de l'intervention un module destiné à l'amélioration de la communication entre les parents et les enfants sur les questions de SR/VIH/SIDA a été conçu. Pour la conception de ce module, le curriculum sur les compétences pour la vie en matière SR/VIH/SIDA (Life Skills) développé par CEDPA et Grandir en Harmonie développé par FRONTIERS. 
L'élaboration de ce curriculum n'a toutefois pas été chose aisée. Le curriculum produit par le consultant était trop théorique, long et complexe pour une utilisation par un personnel peu instruit. Finalement des fiches d'animation ont été tirées du curriculum.

\section{Couverture des thèmes du curriculum}

Dans les activités d'interventions, les thèmes très techniques tels que la connaissance du corps humain, la puberté et l'utilisation de la contraception ont été très peu abordés par les relais. En effet, dans les causeries ces thèmes ont été moins bien traités que les questions de comportement en société, les valeurs, les grossesses désirées etc. Malgré l'organisation d'une journée de réorientation à l'intention des relais parents et adolescent(e)s, le problème est resté.

\section{Faible mobilisation des parents/hommes}

Pour vaincre les réticences des parents et des adolescent(e)s de nombreuses activités de sensibilisation ont été menés. Des foras communautaires et des réunions de plaidoyer ont été organisées dans chacune de ces localités. Ces différentes activités de sensibilisation étaient ouvertes à toute la communauté. Elles ciblaient en particulier les chefs de famille (hommes ou femmes).

Malgré la mobilisation sociale continue et les activités de sensibilisation les hommes étaient faiblement représentés dans les causeries et les sessions de dialogue intergénérationnel. Dans l'ensemble les hommes ne représentaient que 32\% des bénéficiaires parmi les parents.

\section{Durée courte des interventions}

Les activités d'intervention n'ont duré que quelques mois du fait de la fin du projet BRIDGE/CEDPA. La durée très courte de ces interventions n'a donc pas permis de toucher la majorité des parents/tuteurs et des adolescent(e)s.

\section{RÉSULTATS}

\section{Caractéristiques sociodémographiques des adolescent(e)s (tableau 2)}

Profil des adolescent(e)s : L'utilisation des deux échantillons pour mesurer l'évolution des indicateurs attribuables aux interventions suppose que les échantillons présentent suffisamment de similitudes socio-démographiques, c'est-à-dire que le profil des adolescent(e)s interrogé dans les deux phases de l'étude soit similaire. Au-delà de la description des deux échantillons des tests de comparaison ont été effectués pour vérifier la stabilité des 2 échantillons.

Répartition selon l'âge et le sexe : Les proportions d'adolescent(e)s sont restées en général identiques d'une enquête à l'autre. Les deux échantillons montrent une sur représentation des filles (60\%) par rapport aux garçons (40\%). Ceci est probablement dû aux migrations plus importantes des garçons de cet âge pour raison de scolarité ou d'emploi. Entre les deux enquêtes il n'y pas de différence $(\mathrm{p}=0,038)$. L'âge moyen des adolescent(e)s qui ont été interrogés dans les deux phases de l'étude est d'environ 16 ans. Cet indicateur est sensiblement le même dans les deux enquêtes : 15 ans dans l'enquête de base et 16 ans dans l'enquête finale. 
En tenant compte du lieu de résidence des adolescent(e)s, on remarque que si en milieu urbain le profil de l'âge des adolescent(e)s est resté stable entre le moment de l'enquête de base et celui de l'enquête finale (16 ans dans les deux enquêtes) en milieu rural par contre il y a une certaine variation de l'âge. En effet, en milieu rural l'âge des adolescent(e)s passe de 16 ans dans l'enquête de base à 15 ans dans l'enquête finale.

Tableau 2 : Caractéristiques sociodémographiques des adolescent(e)s (en \%)

\begin{tabular}{|c|c|c|c|c|c|c|}
\hline & \multicolumn{2}{|c|}{ Urbain } & \multicolumn{2}{|c|}{ Rural } & \multicolumn{2}{|c|}{ Ensemble } \\
\hline & $\begin{array}{c}\text { Enquête } \\
\text { de base }\end{array}$ & $\begin{array}{c}\text { Enquête } \\
\text { finale }\end{array}$ & $\begin{array}{c}\text { Enquête } \\
\text { de base }\end{array}$ & $\begin{array}{c}\text { Enquête } \\
\text { finale }\end{array}$ & $\begin{array}{c}\text { Enquête } \\
\text { base }\end{array}$ & $\begin{array}{c}\text { Enquête } \\
\text { finale }\end{array}$ \\
\hline $\begin{array}{l}\text { Adolescent(e)s interviewés } \\
\text { Garçons } \\
\text { Filles } \\
\boldsymbol{N} \\
\end{array}$ & $\begin{array}{c}39 \\
61 \\
373 \\
\end{array}$ & $\begin{array}{c}39 \\
61 \\
251 \\
\end{array}$ & $\begin{array}{c}41 \\
59 \\
920 \\
\end{array}$ & $\begin{array}{c}41 \\
59 \\
909 \\
\end{array}$ & $\begin{array}{c}40 \\
60 \\
1293 \\
\end{array}$ & $\begin{array}{c}41 \\
59 \\
1160 \\
\end{array}$ \\
\hline $\begin{array}{l}\text { Age moyen (en années) } \\
\text { Garçons } \\
\text { Filles } \\
\text { Ensemble }\end{array}$ & $\begin{array}{l}16 \\
16 \\
16\end{array}$ & $\begin{array}{l}16 \\
16 \\
16\end{array}$ & $\begin{array}{l}16 \\
16 \\
16 \\
\end{array}$ & $\begin{array}{l}15 \\
15 \\
15\end{array}$ & $\begin{array}{l}16 \\
16 \\
16\end{array}$ & $\begin{array}{l}15 \\
15 \\
15\end{array}$ \\
\hline $\begin{array}{l}\text { Garçons interviewés } \\
10-14 \text { ans } \\
15-19 \text { ans } \\
N\end{array}$ & $\begin{array}{c}20 \\
80 \\
144 \\
\end{array}$ & $\begin{array}{l}22 \\
77 \\
97 \\
\end{array}$ & $\begin{array}{c}26 \\
74 \\
\mathbf{3 7 7} \\
\end{array}$ & $\begin{array}{c}40 \\
60 \\
374 \\
\end{array}$ & $\begin{array}{r}24 \\
76 \\
521 \\
\end{array}$ & $\begin{array}{r}37 \\
64 \\
471 \\
\end{array}$ \\
\hline $\begin{array}{l}\text { Filles interviewées } \\
10-14 \text { ans } \\
15-19 \text { ans } \\
N\end{array}$ & $\begin{array}{c}21 \\
79 \\
229\end{array}$ & $\begin{array}{c}25 \\
75 \\
154 \\
\end{array}$ & $\begin{array}{c}26 \\
74 \\
543 \\
\end{array}$ & $\begin{array}{c}38 \\
62 \\
\mathbf{5 3 5} \\
\end{array}$ & $\begin{array}{c}24 \\
76 \\
772 \\
\end{array}$ & $\begin{array}{c}35 \\
65 \\
689 \\
\end{array}$ \\
\hline $\begin{array}{l}\text { Statut matrimonial } \\
\text { Célibataire } \\
\text { Promis (e) } \\
\text { Marié (e) } \\
N \\
\end{array}$ & $\begin{array}{c}97 \\
1 \\
2 \\
373 \\
\end{array}$ & $\begin{array}{c}97 \\
0 \\
3 \\
251 \\
\end{array}$ & $\begin{array}{c}92 \\
2 \\
6 \\
920 \\
\end{array}$ & $\begin{array}{c}94 \\
2 \\
5 \\
909 \\
\end{array}$ & $\begin{array}{c}94 \\
2 \\
5 \\
1293 \\
\end{array}$ & $\begin{array}{c}94 \\
2 \\
4 \\
1160 \\
\end{array}$ \\
\hline $\begin{array}{l}\text { Activités } \\
\text { Sans occupation } \\
\text { Chômeur } \\
\text { Elève/étudiant } \\
\text { Occupé } \\
N \\
\end{array}$ & $\begin{array}{c}8 \\
1 \\
85 \\
6 \\
373 \\
\end{array}$ & $\begin{array}{c}6 \\
0 \\
84 \\
10 \\
251 \\
\end{array}$ & $\begin{array}{c}22 \\
0 \\
65 \\
13 \\
920\end{array}$ & $\begin{array}{c}11 \\
0 \\
72 \\
17 \\
909\end{array}$ & $\begin{array}{c}18 \\
1 \\
71 \\
11 \\
\mathbf{1 2 9 3} \\
\end{array}$ & $\begin{array}{c}10 \\
0 \\
74 \\
15 \\
1160 \\
\end{array}$ \\
\hline $\begin{array}{l}\text { Niveau d'instruction } \\
\text { Sans instruction } \\
\text { Niveau primaire } \\
\text { Niveau secondaire } \\
\boldsymbol{N} \\
\end{array}$ & $\begin{array}{c}4 \\
30 * \\
66 \\
373 \\
\end{array}$ & $\begin{array}{c}6 \\
21 * \\
73 \\
251 \\
\end{array}$ & $\begin{array}{c}23 \\
50 * \\
26 \\
920 \\
\end{array}$ & $\begin{array}{c}20 \\
44^{*} \\
36 \\
909 \\
\end{array}$ & $\begin{array}{c}18 \\
45^{*} \\
38 \\
\mathbf{1 2 9 3} \\
\end{array}$ & $\begin{array}{c}17 \\
39 * \\
44 * \\
1160 \\
\end{array}$ \\
\hline $\begin{array}{l}\text { Environnement familial l'a } \\
\text { Vit avec les deux parents } \\
\text { Vit avec un de ses parents } \\
\text { Ne vit pas avec ses parents } \\
\text { Total (n) }\end{array}$ & $\begin{array}{c}\operatorname{lescent}(\mathbf{e}) \\
51 \\
29 \\
20 \\
373\end{array}$ & $\begin{array}{c}49 \\
32 \\
19 \\
251\end{array}$ & $\begin{array}{c}58 \\
20 \\
22 \\
920\end{array}$ & $\begin{array}{c}54 \\
21 \\
25 \\
909\end{array}$ & $\begin{array}{c}56 \\
23 \\
22 \\
\mathbf{1 2 9 3}\end{array}$ & $\begin{array}{c}53 \\
23 \\
24 \\
1160\end{array}$ \\
\hline
\end{tabular}

* Différence significative $p<0,1$ 
Le groupe d'âge des 14-15 ans est généralement plus représenté que celui des 10-14 ans et cela aussi bien dans l'enquête de base que dans l'enquête finale. Cette situation a été observée aussi bien chez les garçons que chez les filles.

Situation matrimoniale des adolescent(e)s :La quasi-totalité des adolescent(e)s en milieu rural et en milieu urbain se déclarent célibataires. Les adolescent(e)s séparés de leurs conjoints (pour divorce ou veuvage) sont mêlés aux adolescent(s) mariés du fait le leur nombre très petit.

Niveau d'instruction : Les adolescent(e)s ayant le niveau du primaire représentent la catégorie la plus nombreuse et ceci aussi bien dans l'enquête de base (45\%) que dans l'enquête finale (39\%). Viennent ensuite ceux qui ont atteint le niveau du secondaire (38\% et $44 \%$ ). La proportion des adolescent(e)s n'ayant jamais fréquenté le système scolaire est très faible dans les deux enquêtes. En effet, ce sont respectivement 18\% des adolescent(e)s interrogés dans l'enquête de base et $17 \%$ qui ont déclaré n'avoir jamais été l'école. Aucune différence n'est observée entre les deux enquêtes. Cependant il y a un glissement entre ceux du primaire et secondaire.

Les adolescent(e)s issus du milieu urbain sont nettement plus instruits que ceux qui ont été interrogé en milieu rural. En effet, alors qu'en milieu urbain la proportion des adolescent(e)s n'ayant jamais fréquenté le système scolaire était seulement de $4 \%$ dans l'enquête de base et $6 \%$ dans l'enquête finale (différence non significative, $\mathrm{p}=0,0201$ ), en milieu rural par contre la proportion des adolescent(e)s sans instruction était estimée à $23 \%$ dans l'enquête de base et $20 \%$ dans l'enquête d'évaluation ( $\mathrm{p}=0,041)$.

Religion : La composition de l'échantillon reflète la structure religieuse du pays avec une très large majorité de musulman.

Activité des adolescent(e)s : Dans l'ensemble, plus de la moitié des adolescent(e)s dans les deux enquêtes sont des élèves. Ils représentent la catégorie la plus importante parmi les adolescent(e)s interrogés dans l'enquête de base $(71 \%)$ et dans l'enquête finale $(74 \%)$; viennent ensuite les adolescent(e)s sans occupation (18\% et $10 \%)$ puis les adolescent(e)s se déclarant «occupés» (11\% et $15 \%)$. La proportion des adolescent(e)s ayant un emploi est plus importante en milieu rural $(17 \%)$ qu'en milieu urbain $(10 \%)$.

Personne avec qui vit l'adolescent(e)s : Dans l'ensemble, les adolescent(e)s vivant avec leurs deux parents (le père et la mère) sont plus nombreux (tableau 3). En effet, aussi bien dans l'enquête de base que dans l'enquête d'évaluation, on remarque que plus de la moitié des adolescent(e)s vivent avec leurs deux parents. La proportion de ces enfants est de (56\% et 53\%). La proportion des adolescent(e)s vivant avec l'un ou l'autre des deux parents ne varie pratiquement pas d'une enquête à l'autre $(23 \%)$.

La majorité des adolescent(e)s ayant participé à cette étude ont vécu au cours des cinq dernières années en milieu rural $(70 \%$ et $73 \%)$.

La co-résidence avec les deux parents reste encore le mode de résidence le plus répandu parmi les adolescent(e)s. Cette proportion était estimée à environ $56 \%$ dans l'enquête de base et 53\% dans l'enquête finale. La proportion des adolescent(e)s qui ont déclaré vivre avec l'un ou l'autre des deux parents est pratiquement la même (23\%) dans les deux enquêtes.

A coté des adolescent(e)s vivant avec les deux parents et de ceux qui vivent avec l'un ou l'autre des deux parents, il existe aussi des adolescent(e)s qui sont hébergés par des personnes autres que les parents biologiques : un tuteur ou une tutrice. La proportion de ces adolescent(e)s étant 
plus importante pour les garçons dans les deux enquêtes. Environ 20-23\% des adolescent(e)s déclaraient vivre chez un tuteur ou une tutrice. La forte proportion d'adolescent(e)s vivant avec un tuteur/tutrice s'explique par le fait que dans le contexte sénégalais certains enfants sont très tôt «confiés » à un membre ou à un ami de la famille (Locoh, 1988 ; Savané, 1994 ; Vandermeersch, 2002). Ces enfants se retrouvent souvent dans des réseaux d'hébergement où ils n'en sortent que dans le cas où ils ont leur propre logement.

Tableau 3 : Répartition des adolescent(e)s selon le sexe, le lieu de résidence et la cohabitation avec les parents (en \%)

\begin{tabular}{|c|c|c|c|c|c|c|}
\hline & \multicolumn{2}{|c|}{ Garçons } & \multicolumn{2}{|c|}{ Filles } & \multicolumn{2}{|c|}{ Ensemble } \\
\hline & $\begin{array}{l}\text { Enquête } \\
\text { de base }\end{array}$ & $\begin{array}{c}\text { Enquête } \\
\text { finale }\end{array}$ & $\begin{array}{l}\text { Enquête } \\
\text { de base }\end{array}$ & $\begin{array}{l}\text { Enquête } \\
\text { finale }\end{array}$ & $\begin{array}{l}\text { Enquête } \\
\text { de base }\end{array}$ & $\begin{array}{l}\text { Enquête } \\
\text { finale }\end{array}$ \\
\hline \multicolumn{7}{|l|}{ Lieu de résidence } \\
\hline A vécu en milieu urbain & 30 & 25 & 31 & 29 & 30 & 27 \\
\hline A vécu en milieu rural & 70 & 75 & 69 & 71 & 70 & 73 \\
\hline $\mathrm{N}$ & 520 & 471 & 773 & 689 & 1293 & 1160 \\
\hline \multicolumn{7}{|c|}{ Cohabitation avec les parents } \\
\hline Les deux parents & 59 & 56 & 54 & 51 & 56 & 53 \\
\hline $\begin{array}{l}\text { Pére seul (ou avec un } \\
\text { autre) }\end{array}$ & 4 & 4 & 4 & 3 & 4 & 4 \\
\hline $\begin{array}{l}\text { Mère seule (ou avec un } \\
\text { autre) }\end{array}$ & 19 & 17 & 19 & 21 & 19 & 20 \\
\hline Tuteur / tutrice & 17 & 22 & 22 & 24 & 20 & 23 \\
\hline Autres & 1 & 1 & 2 & 1 & 1 & 1 \\
\hline $\mathbf{N}$ & 521 & 471 & 772 & 689 & 1293 & 1160 \\
\hline
\end{tabular}

En résumé, entre les deux enquêtes le profil des enquêtés a très peu varié. Les échantillons des deux enquêtes sont comparables pour la plupart des caractéristiques socio-démographiques. Les quelques changements observés entre les deux échantillon sont liés au caractère transitoire de l'adolescence, très sensible aux mutations de la société (mariage, migration, scolarité...).

\section{Caractéristiques sociodémographiques des parents}

Ce sont respectivement 896 et 762 parents ou tuteurs d'adolescent(e)s qui ont été interrogés dans l'enquête de base et dans la phase finale de l'étude. Les caractéristiques ci-dessous ont été observées chez les parents/tuteurs d'adolescent(e)s (tableau 4).

Répartition selon le sexe : L'échantillon des parents comprend plus d'hommes que de femmes et cela aussi bien dans la première phase de l'étude que dans la seconde. En effet, dans les deux enquêtes, plus de la moitié des parents/tuteurs sont des femmes (respectivement 68\% et 71\%). Cette différence peut être liée au fait que les hommes étaient souvent absents des ménages lors du passage des enquêteurs, soit de façon temporaire, soit sur une période plus longue.

Répartition selon l'âge : En ce qui concerne l'âge moyen, on remarque que dans l'ensemble les hommes ont une structure par âge plus vieille que les femmes. Quelle que soit la zone de 
résidence l'âge moyen des hommes est d'environ 54 ans alors que pour les femmes l'âge moyen est de 44 ans. Les femmes se déclarant parents/tuteurs seraient donc nettement plus jeunes que les hommes qui se déclarent de ce statut. Il n'existe pratiquement aucune différence entre le milieu urbain et le milieu rural.

Tableau 4 : Répartition des parents selon certaines caractéristiques (en \%)

\begin{tabular}{|c|c|c|c|c|c|c|c|}
\hline & \multicolumn{2}{|c|}{ Urbain } & \multicolumn{2}{|c|}{ Rural } & \multicolumn{2}{|c|}{ Ensemble } \\
\hline & & $\begin{array}{l}\text { Enquête } \\
\text { de base }\end{array}$ & $\begin{array}{c}\text { Enquête } \\
\text { finale }\end{array}$ & $\begin{array}{l}\text { Enquête } \\
\text { de base }\end{array}$ & $\begin{array}{c}\text { Enquête } \\
\text { finale }\end{array}$ & $\begin{array}{l}\text { Enquête } \\
\text { de base }\end{array}$ & $\begin{array}{c}\text { Enquête } \\
\text { finale }\end{array}$ \\
\hline Sexe & $\begin{array}{l}\text { Hommes } \\
\text { Femmes } \\
\mathbf{N}\end{array}$ & $\begin{array}{c}27 \\
73 \\
\mathbf{2 5 2} \\
\end{array}$ & $\begin{array}{c}26 \\
74 \\
\mathbf{1 7 2} \\
\end{array}$ & $\begin{array}{c}34 \\
66 \\
644 \\
\end{array}$ & $\begin{array}{c}30 \\
70 \\
\mathbf{5 9 0} \\
\end{array}$ & $\begin{array}{c}32 \\
68 \\
\mathbf{8 9 6} \\
\end{array}$ & $\begin{array}{c}29 \\
71 \\
\mathbf{7 6 2} \\
\end{array}$ \\
\hline Age moyen & $\begin{array}{l}\text { Hommes } \\
\mathbf{N}\end{array}$ & $\begin{array}{l}53 \\
69 \\
\end{array}$ & $\begin{array}{l}58 \\
44 \\
\end{array}$ & $\begin{array}{c}54 \\
217\end{array}$ & $\begin{array}{c}53 \\
176 \\
\end{array}$ & $\begin{array}{c}54 \\
286 \\
\end{array}$ & $\begin{array}{c}54 \\
220 \\
\end{array}$ \\
\hline (en années) & $\begin{array}{l}\text { Femmes } \\
\mathbf{N}\end{array}$ & $\begin{array}{c}44 \\
\mathbf{1 8 3} \\
\end{array}$ & $\begin{array}{c}46 \\
\mathbf{1 2 8} \\
\end{array}$ & $\begin{array}{c}43 \\
\mathbf{4 2 7} \\
\end{array}$ & $\begin{array}{c}44 \\
\mathbf{4 1 4} \\
\end{array}$ & $\begin{array}{c}43 \\
610 \\
\end{array}$ & $\begin{array}{c}44 \\
\mathbf{5 4 2} \\
\end{array}$ \\
\hline $\begin{array}{l}\text { Niveau } \\
\text { d'instruction }\end{array}$ & $\begin{array}{l}\text { Sans instruction } \\
\text { Primaire } \\
\text { Secondaire et plus } \\
\mathbf{N}\end{array}$ & $\begin{array}{c}21 \\
25 \\
54^{*} \\
\mathbf{2 5 2}\end{array}$ & $\begin{array}{c}27 \\
31 \\
42^{*} \\
\mathbf{1 7 2}\end{array}$ & $\begin{array}{l}65 \\
24 \\
10 \\
\mathbf{6 4 4}\end{array}$ & $\begin{array}{c}65 \\
24 \\
11 \\
\mathbf{5 9 0}\end{array}$ & $\begin{array}{c}53 \\
25 \\
23^{*} \\
896\end{array}$ & $\begin{array}{c}56 \\
26 \\
18^{*} \\
762\end{array}$ \\
\hline
\end{tabular}

* Différence significative $p<0,1$

Niveau d'instruction : Le niveau d'instruction des parents/tuteurs est très faible. En effet, alors que dans l'enquête de base $53 \%$ et $56 \%$ des parents/tuteurs étaient sans instruction. La proportion des parents sans instruction est toutefois plus importante en milieu rural où dans les deux enquêtes plus de la moitié des parents/tuteurs se déclarent de ce niveau d'étude. Le niveau le plus élevé atteint par les parents/tuteurs est généralement celui du primaire : $25 \%$ des parents/tuteurs dans l'enquête de base et $26 \%$ dans l'enquête finale ont atteint ce niveau; au secondaire ceux sont $23 \%$ et $18 \%$ des parents qui ont déclaré avoir atteint ce niveau.

En résumé, l'échantillon est similaire entre les deux enquêtes. Il diffère légèrement pour le niveau d'instruction en milieu urbain. Les femmes représentent la catégorie de la population la plus nombreuse. Cette situation provient du fait que lors de la collecte des données les femmes étaient plus présentes dans les ménages que les hommes. S'y ajoute aussi qu'il existe dans la société sénégalaise un trait de caractère fortement ancré dans les mentalités des populations et qui veut que l'éducation des enfants incombe essentiellement aux femmes.

\section{COMMUNICATION ENTRE PARENTS/TUTEURS EN MATIÈRE DE SR/VIH/SIDA}

La famille est le premier lieu de socialisation des enfants et le cadre par lequel certaines valeurs et normes sont transmises par les parents. Du fait de cette situation, l'étude de l'environnement familial occupe une place importante dans l'identification des attitudes et comportements des adolescent(e)s en matière de SR. 


\section{Perception de la facilité de la communication entre les parents et les adolescent(e)s sur les questions de SR/VIH/SIDA}

La perception de la facilité de communiquer avec leurs parents/tuteurs sur les questions de SR/VIH/SIDA est un bon indicateur pour rendre compte des éventuelles difficultés que rencontre les jeunes pour discuter librement de SR. Les adolescent(e)s qui pensent qu'il est facile de discuter avec les parents/tuteurs sur ces questions sont supposés être plus à l'aise pour établir avec ces derniers des relations de dialogue et sont aussi plus réceptifs aux messages qui leur sont délivrés.

Les entretiens plus approfondis de type qualitatif montrent que la «pudeur » (kersa en wolof) est la principale raison invoquée par les adolescent(e)s pour expliquer les difficultés qu'ils rencontrent pour discuter avec leurs parents des questions de SR. Les propos recueillis auprès cette jeune fille illustrent bien cette situation : "Je me sens très mal à l'aise pour discuter avec mes parents de ces questions. Mon père surtout (... ). Je n'ose même pas imaginer avec ce genre de discussion. D’ailleurs même avec ma mère je me sens toujours très gênée, chaque fois qu'il $m$ 'arrive de discuter avec elle de certaines questions en rapport avec la sexualité (...). Faut dire qu'ici au Sénégal, il n'est jamais facile pour un adolescent(e)s de s'asseoir devant à son père ou sa mère et parler avec eux de préservatifs, sida etc. Nous n'avons pas été éduqué comme cela» (M. Mb., 19 ans).

En plus de la pudeur, certains adolescent(e)s ont également invoqué le manque d'ouverture d'esprit des parents. Ces derniers leur donnant très peu l'opportunité d'avoir ce genre de discussion. En témoigne ces propos d'un garçon «certains parents se mettent très vite sur la défensive et n'ont aucune ouverture d'esprit pouvant susciter un échange entre eux. Pourtant quand ils sortent de chez eux ils communiquent avec les enfants d'autrui mais dés qu'ils sont chez eux ils veulent se montrer très durs et très fermes avec leurs propres enfants »(D. $\mathrm{Nd} ., 16$ ans). Le manque d'ouverture des parents qui est souvent considéré par les adolescent(e)s comme une des raisons pour lesquelles ils ont peur de discuter avec leurs parents se manifeste le plus souvent par la suspicion surtout lorsqu'il s'agit des filles.

Ces dernières préfèrent délibérément ne pas aborder avec leurs parents certains surjets de peur qu'ils ne pensent qu'elles expérimentent les connaissances acquises. «Des fois, il m'arrive d'avoir envie de discuter avec mon père ou ma mère de certaines questions de SR qui me préoccupe (...) Mais vous savez mes parents sont pas faciles. Ma mère surtout. Elle va penser que je suis enceinte ou qu je commence à fréquenter les garçons (...). Vous savez chez nous les parents ne voient pas toujours cela d'un bon oil. Pour eux les questions de sexualité sont tabous » (jeune fille).

Les données quantitatives montrent que pour ce qui est de la perception de la facilité de discuter avec les parents/tuteurs des questions de SR/VIH/SIDA a beaucoup évolué avec les interventions. Les adolescent(e)s qui été interrogés dans la seconde phase de l'étude sont nettement plus nombreux à penser qu'il est facile de discuter avec leurs parents des questions de ce genre et ceci quel que soit le lieu de résidence, l'âge et le sexe des adolescent(e)s (Graphique 1).

La proportion des adolescent(e)s qui considèrent qu'il est facile de discuter librement des questions de SR avec les parents/tuteurs augmente significativement entre les deux enquêtes 
passant de $25 \%$ dans l'enquête de base à environ 38\% dans l'enquête d'évaluation. Soit 13 points d'écart.

La zone de résidence des adolescent(e)s semble être déterminante dans la façon dont les adolescent(e)s perçoivent la facilité de discuter avec les parents sur les questions de SR. Les adolescent(e)s issus du milieu rural étant plus nombreux à penser qu'il est facile de discuter avec les parents. Cette situation a été observée aussi bien dans l'enquête de base que dans l'enquête finale. En milieu rural, la proportion des adolescent(e)s (filles et garçons) qui pensent qu'il est facile de discuter des questions de SR avec les parents est d'environ $26 \%$ dans l'enquête de base et $40 \%$ dans l'enquête finale (Graphique 1). En milieu urbain, la proportion des adolescent(e)s qui déclarent n'éprouver aucune difficulté à discuter avec leurs parents des questions de SR est de $23 \%$ dans l'enquête de base à $28 \%$ dans l'enquête finale. Il n'y pas de changement significatif $(\mathrm{p}=0,173)$. C'est en milieu rural que le changement est significatif (14 points).

\section{Graphique 1 : Répartition (\%) des adolescent(e)s selon la perception par rapport à la facilité de discuter de la SR avec leurs parents, suivant le milieu de résidence}

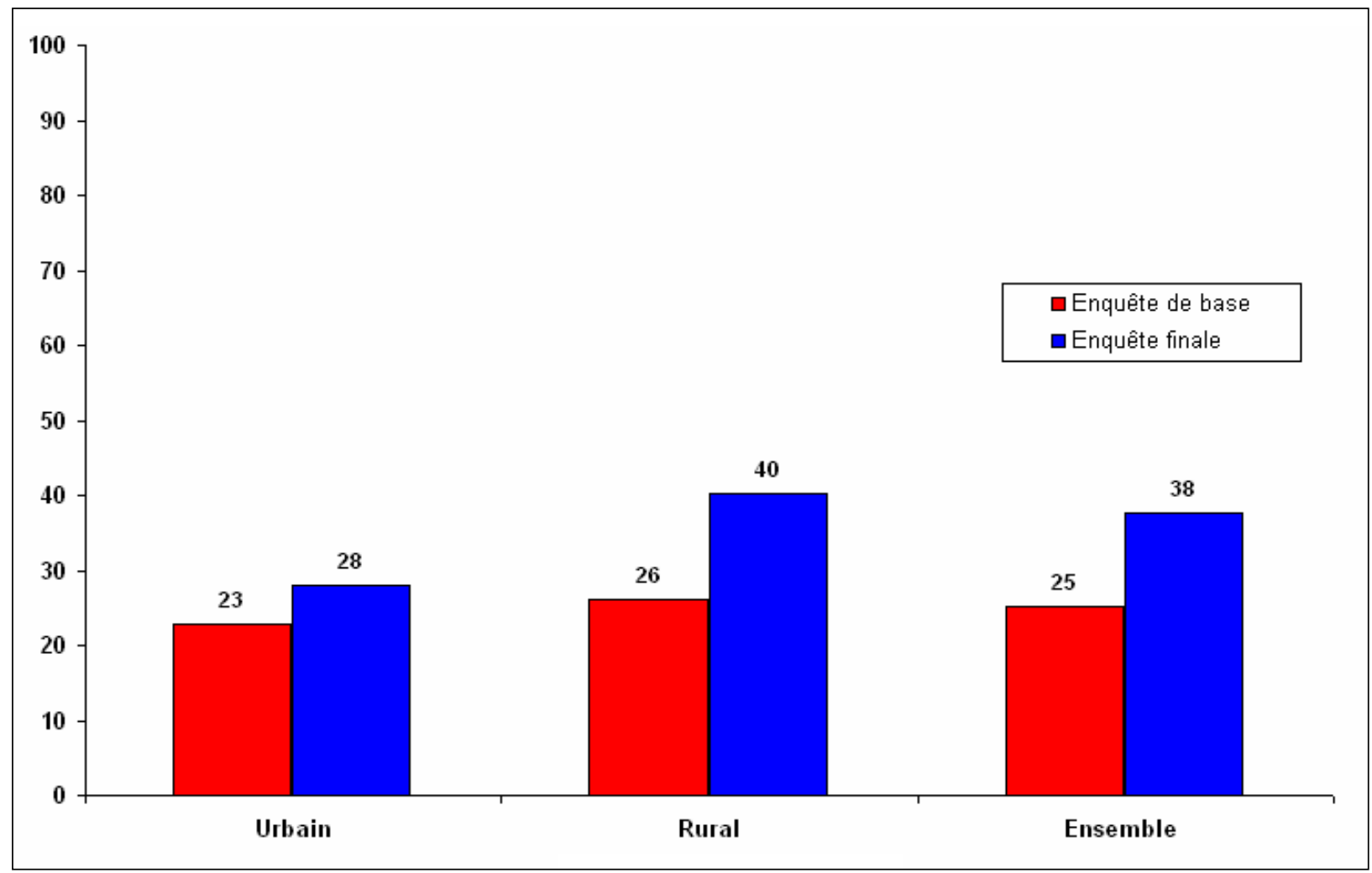

Plus on avance en âge, plus la peur de discuter avec les parents s'estompe. Par rapport à leurs aînés (15-19 ans), les adolescent(e)s les plus jeunes (10-14 ans) sont plus nombreux à considérer qu'il est difficile de discuter avec leurs parents des questions de SR. Cette situation qui a surtout été observée dans l'enquête de base s'est nettement améliorée par la suite (Graphique 2). En effet, dans l'enquête de base seulement 17\% des adolescent(e)s ayant entre 10-14 ans considéraient qu'il était facile de discuter des questions de SR avec les parents/tuteurs, alors que 
pour le groupe des 15-19 ans c'était 28\%. A l'enquête finale tous les deux groupes ont significativement progressé même si l'augmentation est plus forte pour les $10-14$ ans (17\% à $37 \%)$.

Graphique 2 : Répartition ( \%) des adolescent(e)s selon la perception par rapport à la facilité de discuter de la SR avec leurs parents, suivant l'âge et le genre

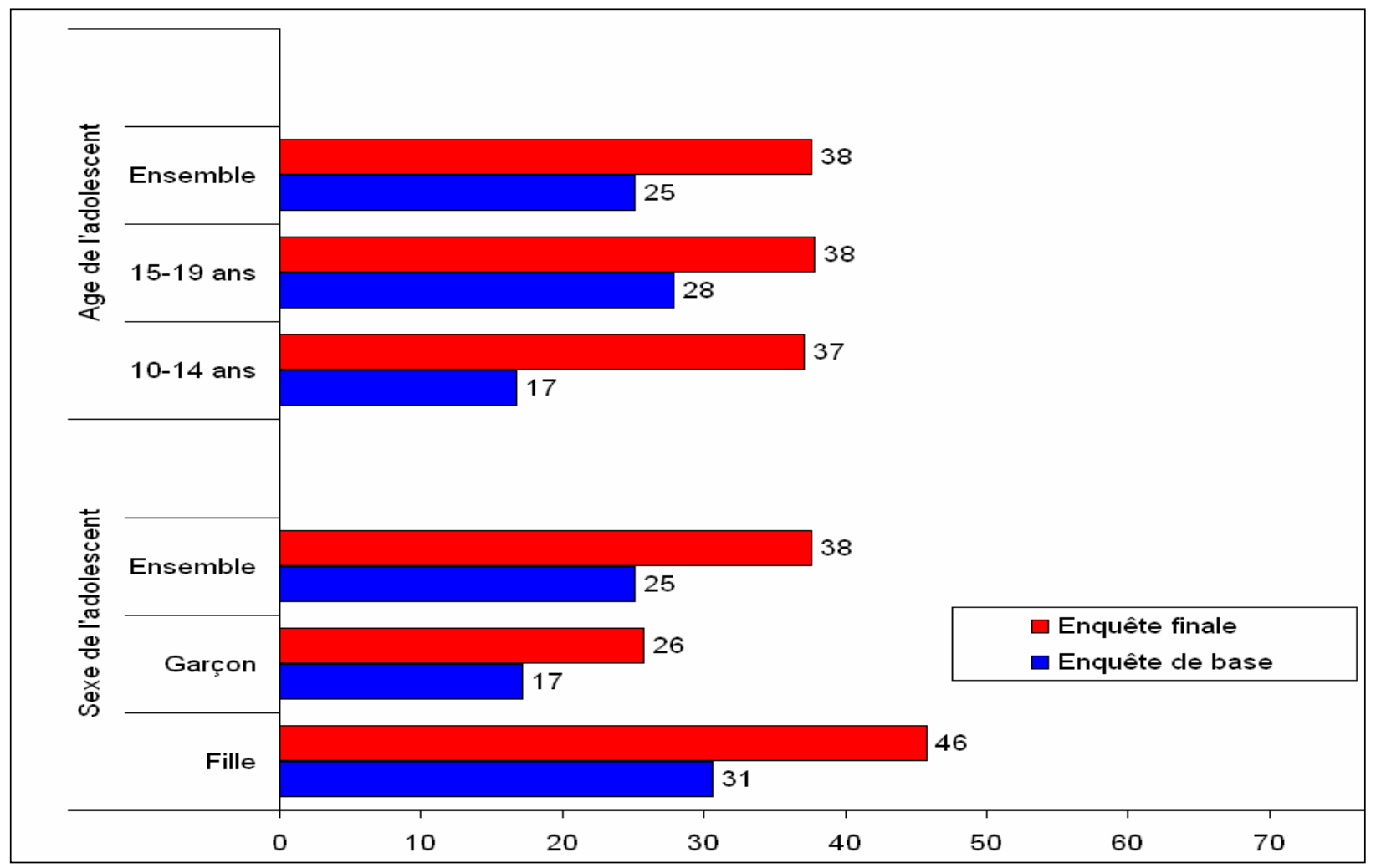

En tenant compte du sexe, la proportion des filles qui déclarent se sentir à l'aise pour discuter des questions de santé de la reproduction avec leurs parents/tuteurs est nettement plus importante que celle des garçons. Cette situation a été observée aussi bien dans l'enquête de base que dans l'enquête finale. En effet les filles ont été plus nombreuses que les garçons à considérer qu'il était facile de discuter des questions de SR avec les parents/tuteurs. Mais pour les deux sexes, il $\mathrm{y}$ a une progression significative.

\section{LE NIVEAU DE LA COMMUNICATION ENTRE PARENTS ET ADOLESCENT(E)S}

Le niveau de la communication entre les parents/tuteurs et les adolescent(e)s en matière de santé de la reproduction est analysé ici à travers la proportion des adolescent(e)s qui déclarent avoir ce genre de discussion avec leurs parents/tuteurs. Dans ce cadre, l'analyse des résultats montre que dans les familles sénégalaises, les parents et les adolescent(e)s ont souvent l'occasion de discuter 
des questions de SR comme en témoigne la forte proportion des adolescent(e)s qui déclarent avoir ce genre de discussion avec leurs parents/tuteurs et ceci quel que soit le lieu de résidence et l'âge des adolescent(e)s.

La proportion des adolescent(e)s qui déclarent communiquer avec leurs parents des questions de SR est nettement plus importante dans l'enquête finale que dans l'enquête de base. Cette proportion a connu une croissance d'environ 5\% entre les deux enquêtes passant de $57 \%$ dans l'enquête de base à environ $62 \%$ dans l'enquête finale $(\mathrm{p}=0,008)$. Ces résultats cachent toutefois de nombreuses disparités selon la zone de résidence des adolescent(e)s.

Alors qu'en milieu rural, la proportion des adolescent(e)s qui déclarent avoir ce genre de discussion avec leurs parents augmente significativement d'une enquête à l'autre $(p=0,004)$, en milieu urbain par contre on ne note pas de changement (Graphique 3). En effet en milieu rural, on passe de $57 \%$ dans l'enquête de base à $63 \%$ et en milieu urbain cela ne change pas $(58 \%$ à $57 \% \mathrm{p}=0,895)$.

\section{Graphique 3 : Répartition (\%) des adolescent(e)s qui déclarent discuter des questions de SR selon le milieu de résidence}

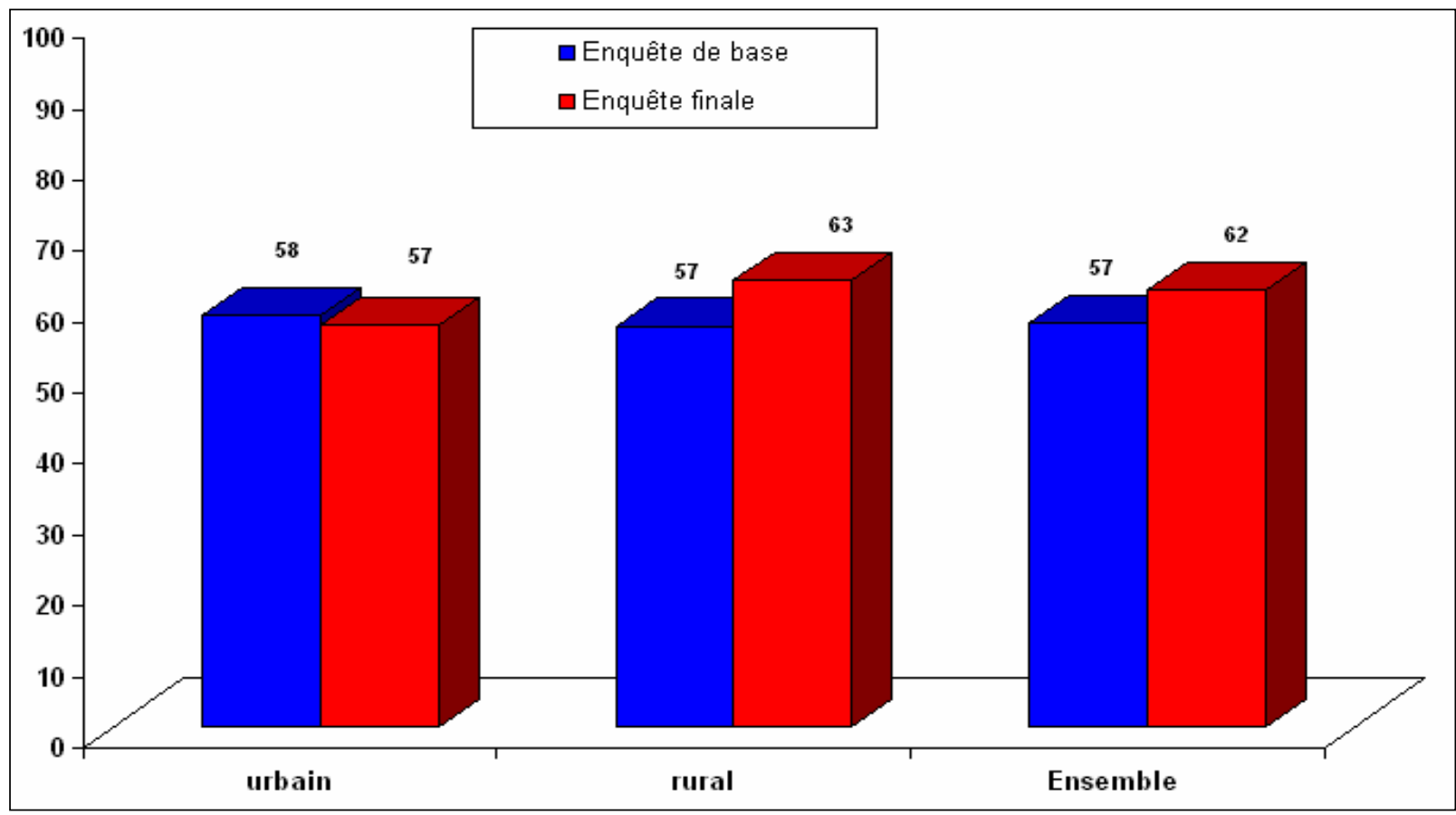

En ce qui concerne le sexe, même si les niveaux de discussion entre les parents/tuteurs et les adolescent(e)s augmentent aussi bien pour les garçons et les filles, il n'en demeure pas moins que les filles sont plus nombreuses à discuter de ces questions que les garçons. Cette situation se maintient durant la deuxième enquête. Ainsi, dans l'enquête de base environ deux tiers des filles ( $72 \%$ ) et $34 \%$ des garçons ont déclaré discuter souvent de certaines questions de SR avec leurs parents. L'écart se maintient dans l'enquête finale où environ $74 \%$ des filles et $44 \%$ des garçons 
ont déclaré discuter souvent des questions de SR avec leurs parents/tuteurs. L'augmentation du niveau de communication n'est significatif que chez les garçons $(p=0,003)$.

\section{Graphique 4 : Répartition (\%) des adolescent(e)s qui déclarent discuter des questions de SR selon le genre et l'âge}

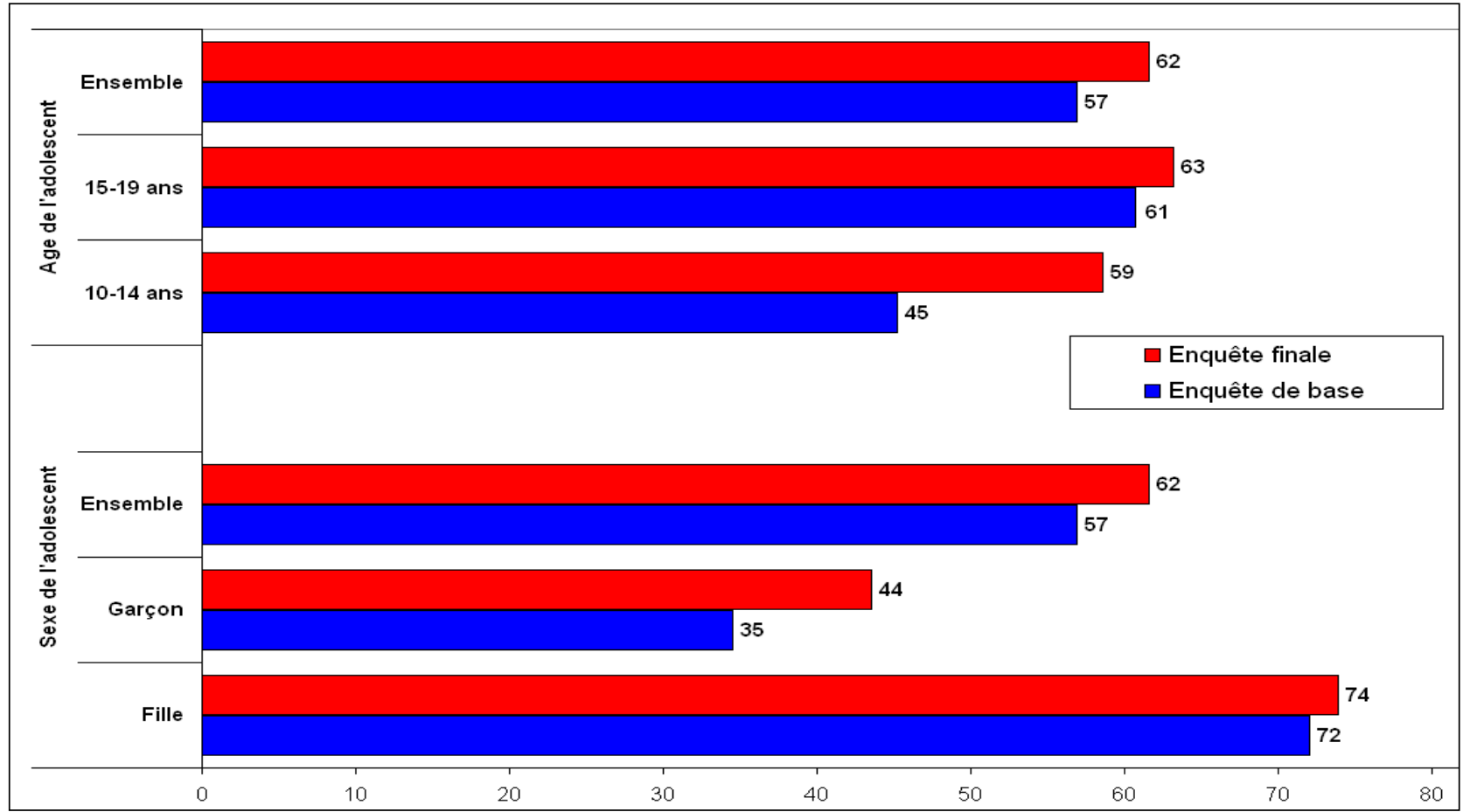

La discussion sur la SR est plus souvent instaurée avec les plus âgés (15-19 ans). Cette situation qui avait été observée dans l'enquête de base se maintient dans le temps même si, par ailleurs, l'écart entre les deux groupes d'âges s'atténue entre les deux enquêtes. En effet, cette proportion pour les 15-19 ans est de $61 \%$ et pour les 10-14 ans de $45 \%$. C'est pour le groupe d'âge 10-14 ans que les interventions ont eu des effets significatifs ( $45 \%$ à $59 \% \mathrm{p}=0,000)$.

En résumé, les parents/tuteurs jouent un grand rôle dans l'éducation sexuelle des adolescent(e)s. Car les niveaux sont déjà élevés au départ. Cette éducation se fait, le plus souvent à travers des discussions sur certaines questions en rapport avec la sexualité et la SR. Pour ce faire, les parents ont souvent l'opportunité de discuter avec leurs enfants de ces questions. Cette discussion s'adresse surtout aux adolescent(e)s les plus âgés (15-19 ans) et aux filles. Mais les interventions ont réussi à augmenter positivement la discussion avec les adolescent(e)s plus jeunes et les garçons.

Les raisons invoquées par les parents pour expliquer la nécessité de discuter plus avec les filles qu'avec les garçons sont le plus souvent liées au contrôle de la sexualité des jeunes filles avant le mariage et à l'importance de la virginité dans la société sénégalais. Ces propos recueillis auprès d'une mère de famille illustre bien l'attitude des parents en ce qui concerne la communication en matière de SR: "On doit discuter d'avantage avec les filles des questions de santé de la 
reproduction parce qu'elles sont plus vulnérables que les garçons (...) En plus, c'est très important pour nous les mères que nos filles restent vierges jusqu'au jour du mariage. C'est le plus cadeau qu'elles peuvent offrir à leurs futurs époux et à nous aussi les mères...Quand une fille n'a pu garder sa virginité on dit toujours que c'est la faute de sa mère et portant nous n'arrêtons pas de leurs donner des conseils »

\section{LA QUALITÉ DE LA COMMUNICATION ENTRE ADOLESCENT(E)S ET PARENTS}

\section{Prise d'initiative de la discussion}

La communication est supposée de bonne qualité et non directive lorsque l'initiative ne vient pas toujours et exclusivement des parents/tuteurs. Autrement dit, lorsque les adolescent(e)s ont euxmême la possibilité d'interpeller et discuter avec leurs parents/tuteurs sur un sujet qu'ils auront choisis.

Dans le contexte sénégalais l'initiative de discuter des questions de SR revient dans la plupart des cas aux parents/tuteurs. Les adolescent(e)s ont très peu la possibilité d'interpeller leurs parents sur un sujet de SR qui les préoccupe et d'en discuter librement avec eux. Ainsi, quel que soit l'enquête la proportion des adolescent(e)s qui déclarent que l'initiative de la discussion revient exclusivement à leurs parents est élevé (88\% et $84 \%$ ).

Graphique 5 : Répartition des adolescents qui discutent de SR avec les parents, suivant la personne qui prend l'initiative de la discussion et le lieu de résidence

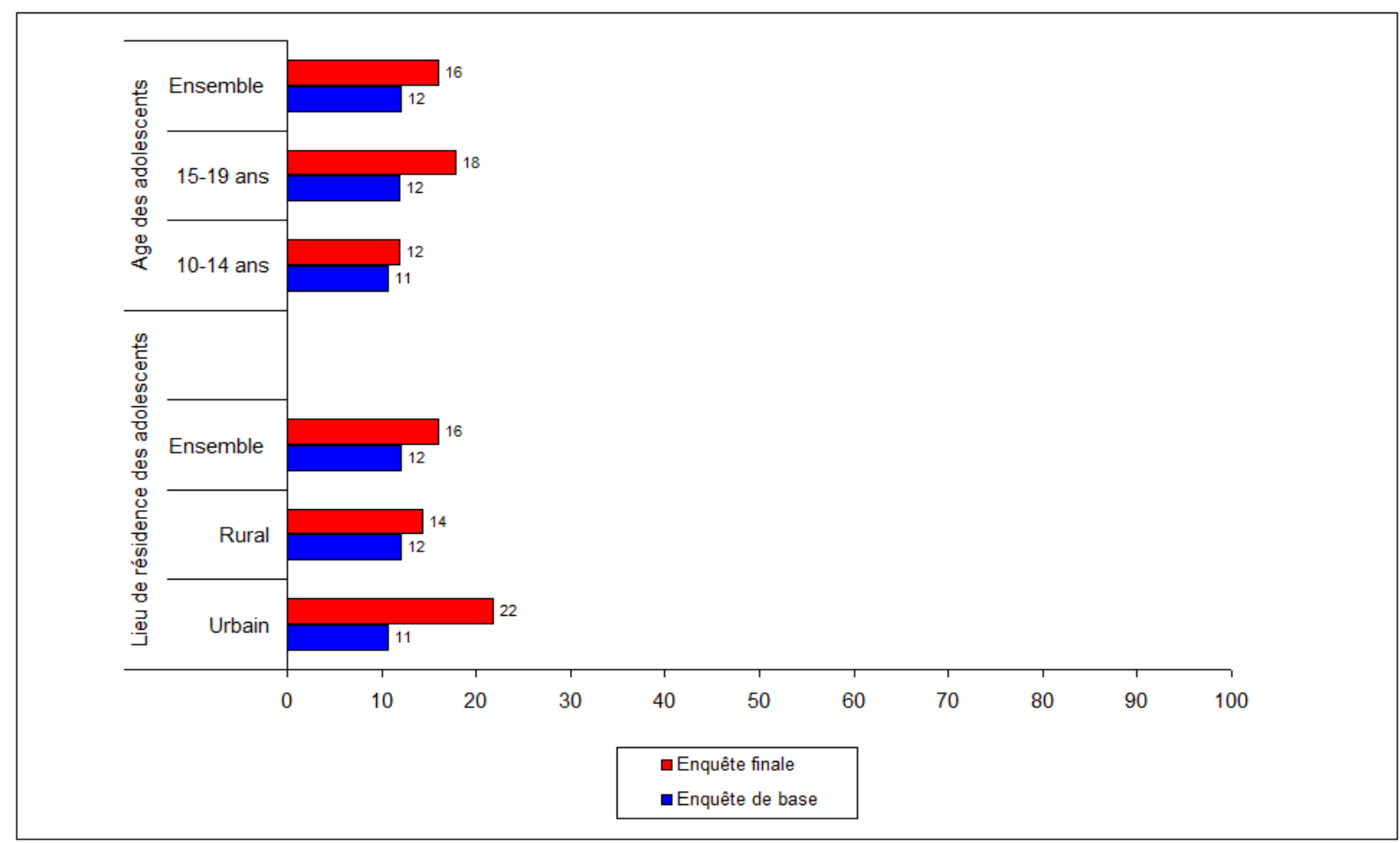


La proportion des adolescent(e)s qui déclarent prendre eux même l'initiative de la discussion a augmenté dans la seconde phase de l'étude même si, par ailleurs, cette augmentation n'est significative qu'en milieu urbain $(\mathrm{p}=0,000)$ ou cela a doublé (Graphique 5).

La proportion des garçons qui déclarent avoir la possibilité de prendre l'initiative de la discussion ne varie pratiquement pas entre les deux enquêtes (14\% dans l'enquête de base et $15 \%$ dans l'enquête finale). Pour les filles cette possibilité de prendre l'initiative de discuter avec leurs parents/tuteurs sur les questions de SR est nettement plus importante dans l'enquête finale (11\% â 17\%). Selon l'âge c'est pour les 15-19 ans que la progression est significative (graphique 6).

\section{Graphique 6 : Répartition des adolescents qui discutent de SR avec les parents, suivant la personne qui prend l'initiative de la discussion, le genre et l'âge}

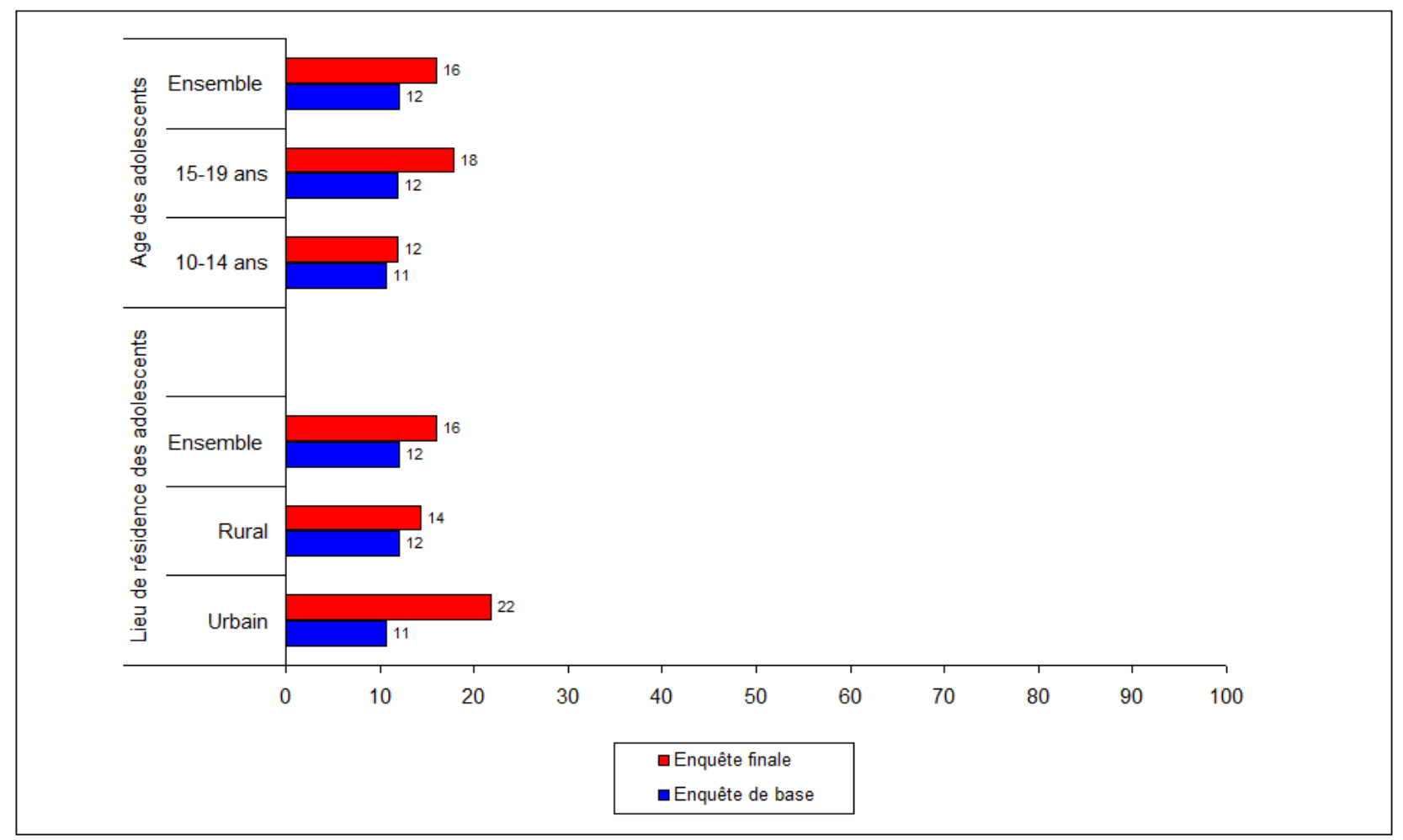

En résumé, si les parents et les adolescent(e)s discutent des questions de SR, il n'en demeure pas moins que l'initiative de cette discussion revient le plus souvent aux parents. Les adolescent(e)s ayant très peu la possibilité de l'initier, surtout pour les plus jeunes et les garçons. Cette faible proportion d'adolescent(e)s qui prennent l'initiative de la discussion résulte sans nul doute des modes de socialisation en vigueur au Sénégal. En effet, la façon dont les filles et les garçons sont éduqués au Sénégal ne leur permet pas toujours prendre l'initiative de discuter avec leurs parents/tuteurs comme en témoigne les propos de cette adolescent(e)se : «ce sont nos parents eux-même qui nous appellent pour discuter en nous réunissant en groupe parce qu'il n'est pas de coutume dans nos sociétés qu'un enfant interpelle ses parent lui-même et prend l'initiative de la discussion » (M. T., 18 ans). 
Ce point de vue est largement partagé par les adolescent(e)s avec qui nous avons discuté. Un adolescent de Sibassor répondait en ces termes «je n'ose pas prendre l'initiative de discuter avec mes parents, ils me tueraient si je le faisais. Ils penseront que j'ai quelque chose en tête ou que je leur manque de respect. Dans tous les cas, il est très rare de voir ici un jeune qui prend lui-même l'initiative de discuter avec ses parents des questions de sexualité. Ce n'est pas dans nos coutumes»(P.D. 15 ans).

Les interventions ont permis d'améliorer significativement la situation chez les adolescent(e)s du milieu urbain et celle des filles.

\section{L'opportunité de poser des questions lors de la discussion}

Le manque d'opportunité pour les adolescent(e)s de poser des questions à leurs parents lorsqu'ils discutent des questions de SR peut être interprété comme une absence de dialogue et donc comme une communication de qualité faible. L'examen des données montre qu'il existe des variations entre les deux enquêtes. La proportion des adolescent(e)s qui déclarent avoir l'opportunité des poser des questions à leurs parents/tuteurs lorsqu'ils discutent des questions de SR étant significativement plus importante dans l'enquête finale que dans l'enquête de base (Graphique 7).

Graphique 7: Répartition des adolescent(e)s selon qu'ils aient ou non l'opportunité de poser des questions lors des discussions avec leurs parents/tuteurs (en \%)

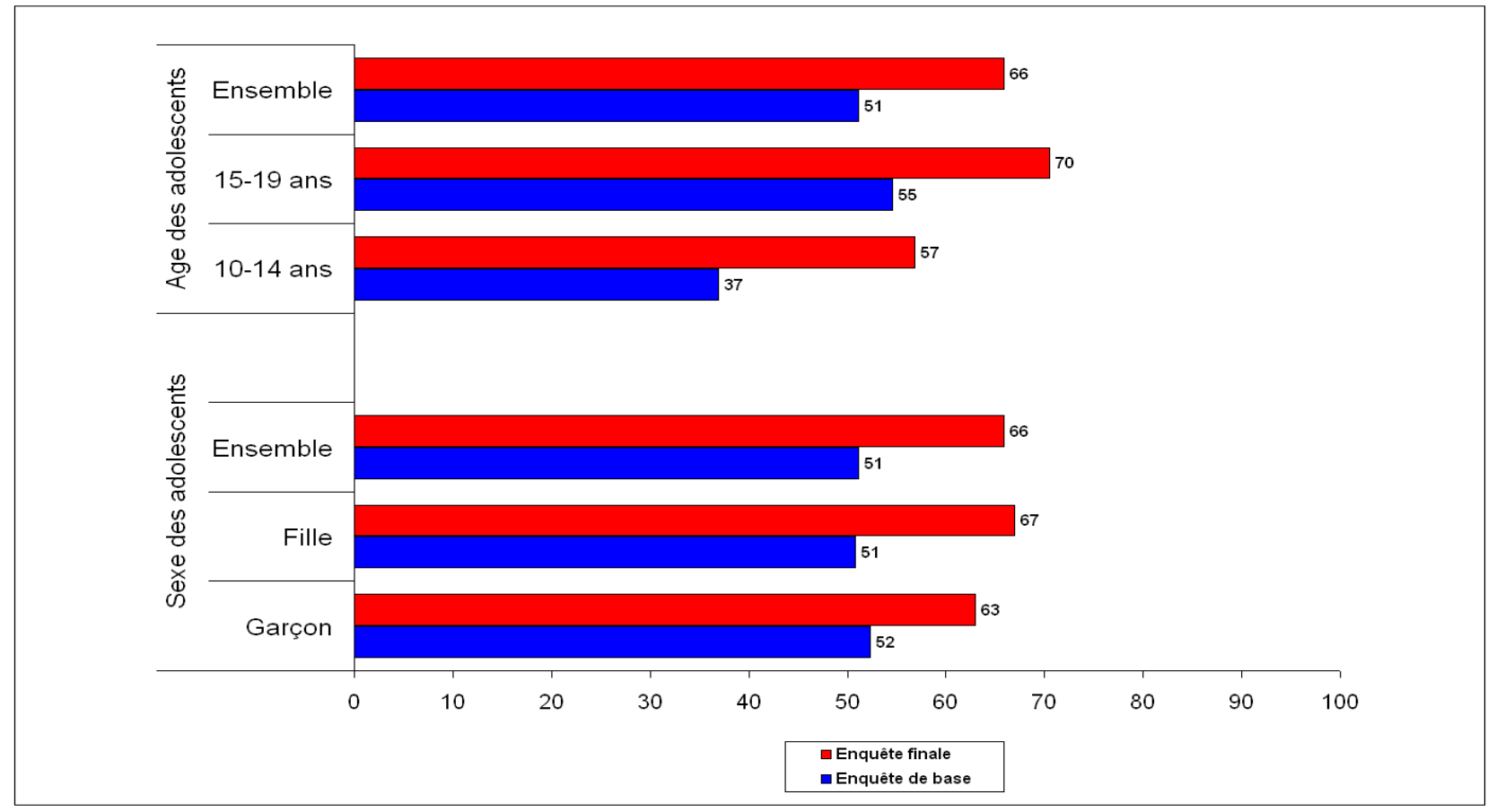

Alors que dans l'enquête de base seulement un peu plus de la moitié (54\%) des adolescent(e)s ont l'opportunité de poser des questions lorsqu'ils discutent avec leurs parents/tuteurs de 
certaines questions de SR, dans l'enquête finale par contre cette proportion est d'environ $66 \%$. Soit une augmentation d'environ 12 points entre les deux enquêtes $(p=0,000)$.

En tenant compte du sexe, si dans l'enquête de base une proportion quasi similaire de filles $(53 \%)$ et de garçons (57\%) étaient concernés par ce comportement, dans l'enquête finale par contre ceux sont $63 \%$ des filles et $67 \%$ des garçons qui déclarent avoir l'opportunité de poser des questions à leurs parents lorsqu'ils discutent des questions de SR. Donc une augmentation significative pour les 2 sexes $(\mathrm{p}=0,000)$.

Pour ce qui est de l'âge des adolescent(e)s, aussi bien dans l'enquête de base que dans l'enquête finale, la proportion des adolescent(e)s qui déclarent avoir l'opportunité de poser des questions à leurs parents/tuteurs lorsqu'ils discutent est plus importante chez les adolescent(e)s les plus âgés que les adolescent(e)s les plus jeunes.

Dans l'enquête finale c'est $57 \%$ pour les adolescent(e)s ayant entre 10-14 ans et 70\% pour les adolescent(e)s ayant entre 15-19 ans contre 37\% dans le groupe des adolescent(e)s ayant entre 10-14 ans et 58\% dans le groupe des adolescent(e)s ayant entre 15-19 ans au départ. Donc une nette progression pour les deux groupes $(\mathrm{p}=0,000)$.

Les interventions ont permis d'améliorer la situation quelque soit les caractéristiques d'âge, de sexe et de résidence.

\section{Réaction des parents lorsque les adolescent(e)s leur posent des questions}

La réaction des parents lorsque les adolescent(e)s leur posent des questions traduit leur ouverture ou non à enclencher une relation de dialogue avec leurs enfants. Dans ce cadre, la communication entre les parents et les adolescent(e)s est supposée de bonne qualité lorsque la réaction des parents est positive c'est à dire lorsque les adolescent(e)s posent des questions à leurs parents/tuteurs et que la réaction de ces derniers suscite l'envie des adolescent(e)s de continuer la discussion. Le tableau 5 donne la répartition des adolescent(e)s, garçons comme filles, selon la réaction de leurs parents lorsqu'ils leurs posent des questions durant les discussions.

Dans l'ensemble, les parents/tuteurs sont plutôt contents lorsqu'ils sont interpellés par les adolescent(e)s et qu'ils doivent répondre à certaines questions qui leur sont posées. En effet, dans les deux enquêtes, la quasi-totalité des adolescent(e)s déclare que leurs parents sont plutôt contents de répondre aux questions qu'ils leurs posent. La proportion de ces adolescent(e)s est d'environ $83 \%$ dans l'enquête de base et $94 \%$ dans l'enquête finale $(p=0.048)$. Très peu de parents/tuteurs réagissent négativement lorsque leurs enfants leur posent des questions de SR. Cette situation qui a été observée dans les deux enquêtes s'est nettement accentué dans la phase finale du programme. Dans ce cadre, on note que si dans l'enquête de base ceux sont seulement $7 \%$ des adolescent(e)s qui déclarent que leurs parents/tuteurs étaient surpris lorsqu'ils leurs posent des questions de SR, dans l'enquête d'évaluation par contre cette réaction est quasi-nulle après. 
Tableau 5 : Répartition des garçons et filles selon la réaction des parents à leurs questions lors des discussions (en \%)

\begin{tabular}{|c|c|c|c|c|c|c|}
\hline & \multicolumn{2}{|c|}{ Garçons } & \multicolumn{2}{|c|}{ Filles } & \multicolumn{2}{|c|}{ Ensemble } \\
\hline & $\begin{array}{l}\text { Enquête } \\
\text { de base }\end{array}$ & $\begin{array}{c}\text { Enquête } \\
\text { finale }\end{array}$ & $\begin{array}{l}\text { Enquête } \\
\text { de base }\end{array}$ & $\begin{array}{l}\text { Enquête } \\
\text { finale }\end{array}$ & $\begin{array}{l}\text { Enquête } \\
\text { de base }\end{array}$ & $\begin{array}{c}\text { Enquête } \\
\text { finale }\end{array}$ \\
\hline Ils sont surpris & 10 & 0 & 6 & 0 & 7 & 0 \\
\hline Ils sont contents de répondre aux questions & 79 & $91^{*}$ & 84 & $94 *$ & 83 & $94^{*}$ \\
\hline Ils me renvoient à d'autres personnes & 1 & 1 & 1 & 1 & 1 & 1 \\
\hline Ils sont gênés, mais répondent & 2 & 0 & 3 & 0 & 3 & 0 \\
\hline Ils réagissent négativement & 5 & 4 & 2 & 4 & 3 & 4 \\
\hline Autre & 3 & 4 & 3 & 1 & 3 & 2 \\
\hline Total & 100 & 100 & 100 & 100 & 100 & 100 \\
\hline \multirow[t]{3}{*}{$N$} & 91 & 129 & 280 & 341 & 372 & 470 \\
\hline & \multicolumn{2}{|c|}{$10-14$ ans } & \multicolumn{2}{|c|}{ 15-19 ans } & \multicolumn{2}{|c|}{ Ensemble } \\
\hline & $\begin{array}{l}\text { Enquête } \\
\text { de base }\end{array}$ & $\begin{array}{c}\text { Enquête } \\
\text { finale }\end{array}$ & $\begin{array}{l}\text { Enquête } \\
\text { de base }\end{array}$ & $\begin{array}{c}\text { Enquête } \\
\text { finale }\end{array}$ & $\begin{array}{l}\text { Enquête } \\
\text { de base }\end{array}$ & $\begin{array}{c}\text { Enquête } \\
\text { finale }\end{array}$ \\
\hline Ils sont surpris & 10 & 0 & 7 & 0 & 7 & 0 \\
\hline Ils sont contents de répondre aux questions & 79 & $91 *$ & 84 & $95^{*}$ & 83 & $94 *$ \\
\hline Ils me renvoient à d'autres personnes & 0 & 0 & 1 & 1 & 1 & 1 \\
\hline Ils sont gênés, mais répondent & 0 & 0 & 3 & 0 & 3 & 0 \\
\hline Ils réagissent négativement & 8 & 6 & 1 & 4 & 3 & 4 \\
\hline Autre & 4 & 2,17 & 3 & 2 & 3 & 2 \\
\hline Total & 100 & 100 & 100 & 100 & 100 & 100 \\
\hline$N$ & 52 & 138 & 320 & 332 & 372 & 470 \\
\hline
\end{tabular}

En tenant compte du sexe, les filles étaient plus nombreuses à partager ce point de vue. Dans l'enquête finale par contre les proportions des filles et de garçons qui déclarent que leurs parents sont contents de répondre aux questions sont quasi-identiques. L'écart entre les deux sexes se résorbe progressivement dans l'enquête finale. Ainsi, alors que dans l'enquête de base $84 \%$ des filles et $79 \%$ des garçons notent une réaction positive des parents/tuteurs lorsqu'ils leurs posent des questions de SR dans l'enquête d'évaluation par contre ceux sont $94 \%$ des filles et $91 \%$ des garçons qui partagent ce point de vue. Mais il y a une progression significative pour les deux.

En résumé, les trois indicateurs qui ont été retenus pour rendre compte de la qualité de la communication entre parents/tuteurs en matière de SR/VIH/SIDA montrent une nette amélioration avec les interventions. Même si l'initiative de la discussion revient toujours quasiexclusivement aux parents/tuteurs, il n'en demeure pas moins qu'entre les deux enquêtes, il y a une augmentation progressive de la proportion des adolescent(e)s qui déclarent prendre euxmême l'initiative de la discussion, surtout pour les adolescents plus âgés. Par ailleurs, les parents sont de plus en plus ouverts à la discussion et de plus en plus nombreux à accepter que leurs enfants leurs posent des questions lorsque l'occasion leur est donné de discuter de SR Mais pour un adolescent qui vit en milieu urbain, ou qui est un garçon, ou qui est très jeune, le dialogue est plus difficile. 


\section{LA FRÉQUENCE DE LA COMMUNICATION}

Par la fréquence de la discussion c'est la régularité de la discussion entre les parents/tuteurs et les adolescent(e)s sur les questions de santé de la reproduction qui est analysée. Les adolescent(e)s ont de plus en plus l'occasion de discuter avec leurs parents/tuteurs des questions de SR.

Ces discussions sont de plus en plus fréquentes dans les différents sites de l'étude. En témoigne l'augmentation, d'une enquête à l'autre, de la proportion des adolescent(e)s qui déclarent discuter souvent de ces questions. Si dans l'enquête de base seulement $42 \%$ des adolescent(e)s ont déclaré discuter souvent des questions de SR avec leurs parents/tuteurs, dans l'enquête finale plus de la majorité $(51 \%)$ des adolescent(e)s sont concernés $(\mathrm{p}=0,001)$.

Les adolescent(e)s qui déclarent discuter occasionnellement de ces questions sont de moins en moins nombreux (40\% à $14 \%$ ). La baisse progressive de la proportion des adolescent(e)s qui discutent occasionnellement de ces questions apparaît comme étant une conséquence des interventions. En effet, l'amélioration de la fréquence de la discussion était un volet important de cette étude (Graphique 8). Il y a une augmentation positive pour les deux sexes.

Selon l'âge, il apparaît que c'est surtout les 10-14 ans qui ont déclaré en plus grand nombre qu'ils communiquaient souvent avec les parents lors de l'enquête finale.

En résumé, les interventions ont pu augmenter significativement la fréquence de la communication.

Graphique 8: Répartition des adolescents selon la fréquence de la discussion avec les parents/tuteurs (en \%)

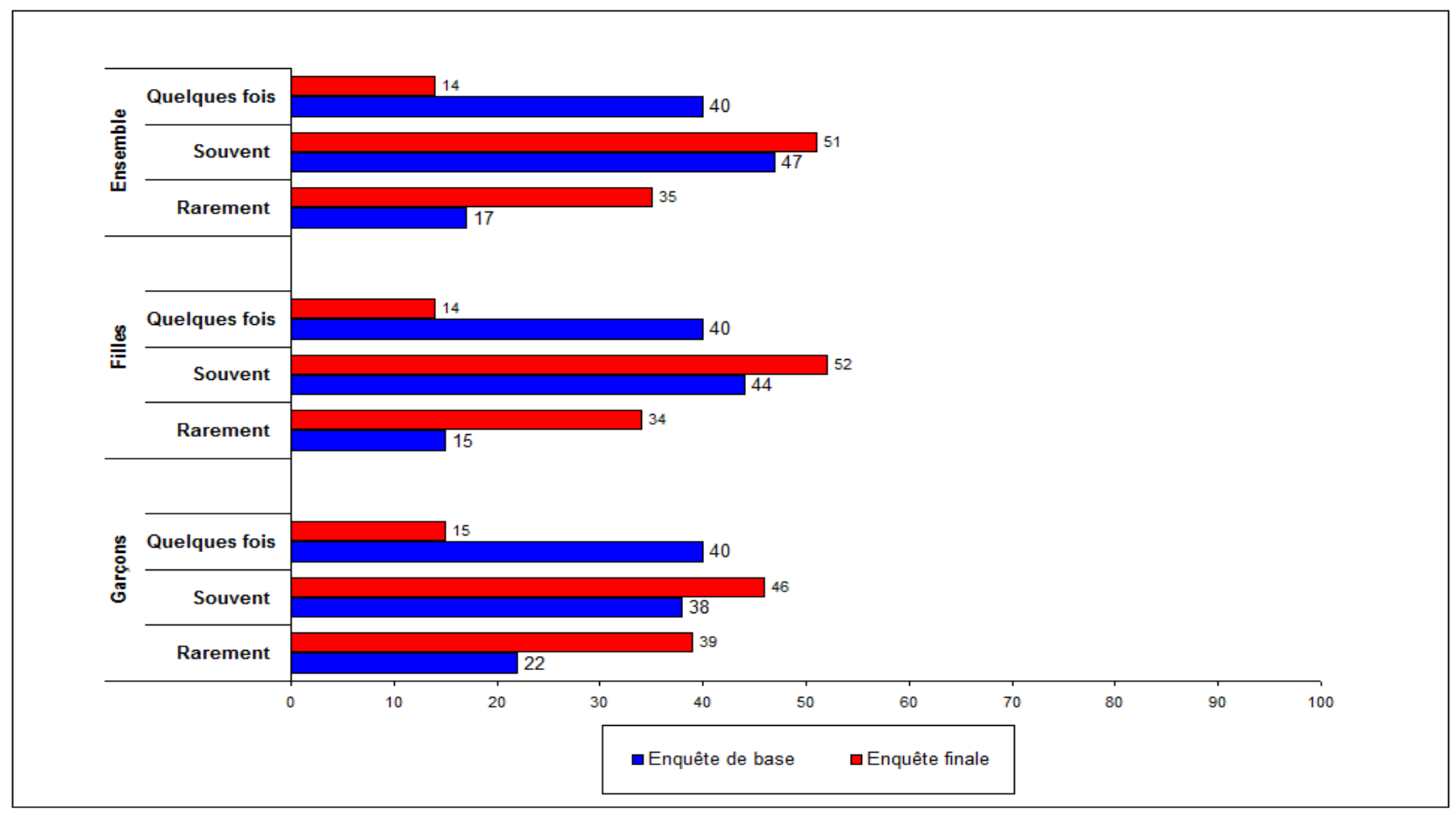




\section{LES THÈMES ABORDÉS LORS DE LA DISCUSSION}

L'examen des informations recueillies auprès des adolescent(e)s montre que dans l'ensemble les thèmes qui sont le plus souvent abordés par les parents/tuteurs et les adolescent(e)s lorsqu'ils discutent des questions de SR varient très peu entre les deux enquêtes. Les thèmes de discussion étant pratiquement les mêmes d'une enquête à l'autre. Dans l'ensemble, ces thèmes sont le plus souvent liés aux comportements des adolescent(e)s dans la société, aux grossesses non désirées et l'abstinence et aux IST/SIDA (Tableau 6).

Inversement certains aspects de la SR sont très peu abordés par les parents/tuteurs et les adolescent(e)s lorsqu'ils discutent de questions de SR/VIH/SIDA. Il s'agit de l'utilisation des méthodes contraceptives modernes, la connaissance du corps humain et à la puberté.

Les thèmes que les parents/tuteurs abordent avec leurs enfants lorsqu'ils discutent des questions de SR sont le plus souvent fonction du sexe des adolescent(e)s. En effet, si pour les garçons c'est les IST/SIDA et les comportements, avec les filles les parents discutent plus des questions liées aux comportements en société et aux grossesses non désirées. Autrement dit, alors que pour les garçons les parents préfèrent mettre l'accent sur les infections et le comportement en société; Pour les filles par contre il semble que l'objectif que visent les parents/tuteurs en discutant avec elles est d'éviter les grossesses non désirées avec un seul moyen qui est l'abstinence et la perte de la virginité.

Tableau 6 : Répartition (\%) des adolescent(e)s selon les aspects de la SR abordés lors des discussions avec les parents selon le sexe

\begin{tabular}{|l|cc|cc|cc|}
\hline \multirow{2}{*}{ Les thèmes abordés lors de la discussion } & \multicolumn{2}{|c|}{ Garçons } & \multicolumn{2}{c|}{ Filles } & \multicolumn{2}{c|}{ Ensemble } \\
\cline { 2 - 7 } & $\begin{array}{c}\text { Enquête } \\
\text { de base }\end{array}$ & $\begin{array}{c}\text { Enquête } \\
\text { finale }\end{array}$ & $\begin{array}{c}\text { Enquête } \\
\text { de base }\end{array}$ & $\begin{array}{c}\text { Enquête } \\
\text { finale }\end{array}$ & $\begin{array}{c}\text { Enquête } \\
\text { de base }\end{array}$ & $\begin{array}{c}\text { Enquête } \\
\text { finale }\end{array}$ \\
\hline Valeurs & 36 & 44 & 42 & 38 & 41 & 40 \\
Estime de soi & 8 & 13 & 16 & 15 & 14 & 15 \\
Comportement & 55 & $72^{*}$ & 57 & $69^{*}$ & 57 & $70^{*}$ \\
Puberté & 3 & 4 & 9 & 10 & 8 & 8 \\
Connaissance du corps & 2 & 1 & 6 & 4 & 5 & 3 \\
Conseil sur l'hygiène & 5 & 1 & 17 & 9 & 14 & 6 \\
Abstinence & 25 & $36^{*}$ & 37 & 43 & 34 & 40 \\
Infos sur les IST/SIDA & 48 & 36 & 21 & 21 & 27 & 26 \\
Eviter les grossesses non désirées & 28 & 27 & 51 & 52 & 45 & 45 \\
Contraception & 1 & 2 & 2 & 5 & 2 & 3 \\
N & $\mathbf{1 7 4}$ & $\mathbf{2 0 5}$ & $\mathbf{5 5 6}$ & $\mathbf{5 0 9}$ & $\mathbf{7 3 1}$ & $\mathbf{7 1 4}$ \\
\hline
\end{tabular}

\section{ATTITUDE DES ADOLESCENT(E)S VIS-À-VIS DES PROGRAMMES D'AMÉLIORATION DE LA COMMUNICATION}

De manière générale, les adolescent(e)s ont une attitude très positive quant à la nécessité d'améliorer et de renforcer la communication avec leurs parents/tuteurs. En effet, pour les adolescent(e)s les parents/tuteurs devraient d'avantage prendre en charge leurs besoins en 
matière d'informations sur les questions de SR et discuter avec eux plus souvent de ces questions. Ce point de vue est partagé par la quasi-totalité des adolescent(e)s qui ont été interrogés dans l'enquête de base (95\%) et l'enquête finale (96\%).

Comme l'indique le tableau 7, le comportement en société, les informations sur les IST/SIDA, les grossesses non désirées et les valeurs sont les aspects que les adolescent(e)s ont le plus cités comme ceux qu'ils voudraient aborder avec leurs parents. Chez les garçons, ce sont les sujets liés aux informations sur les IST/SIDA et au comportement en société qui sont les plus évoqués tandis que les filles aimeraient plus aborder à l'avenir avec leurs parents des questions relatives aux grossesses non désirées, aux comportements en société, aux informations sur les IST/SIDA et aux valeurs d'abstinence et de virginité. Il faut noter qu'après les interventions les adolescent(e)s aimeraient discuter plus de tous les aspects de la SR, de la puberté et éviter les grossesses.

Tableau 7: Répartition (\%) des adolescent(e)s selon qu'ils aimeraient ou non que les parents discutent plus avec eux des questions liées à la SR selon l'âge et le sexe

\begin{tabular}{|l|cc|cc|cc|}
\hline & \multicolumn{2}{|c|}{ Garçons } & \multicolumn{2}{|c|}{ Filles } & \multicolumn{2}{c|}{ Ensemble } \\
\cline { 2 - 6 } & $\begin{array}{c}\text { Enquête } \\
\text { de base }\end{array}$ & $\begin{array}{c}\text { Enquête } \\
\text { finale }\end{array}$ & $\begin{array}{c}\text { Enquêt } \\
\text { e de } \\
\text { base }\end{array}$ & $\begin{array}{c}\text { Enquête } \\
\text { finale }\end{array}$ & $\begin{array}{c}\text { Enquête } \\
\text { de base }\end{array}$ & $\begin{array}{c}\text { Enquête } \\
\text { finale }\end{array}$ \\
\hline Désire aborder le plus souvent les questions de & & & & & \\
SR & 93 & 96 & 95 & 97 & 95 & 96 \\
Aspects à aborder avec les parents/tuteurs & & & & & \\
Tous les aspects de la SR & 16 & $22^{*}$ & 20 & $35^{*}$ & 19 & $30^{*}$ \\
Comportement en société & 47 & 51 & 45 & 48 & 46 & 49 \\
Valeurs & 23 & 24 & 30 & 27 & 27 & 25 \\
Conseil sur l'hygiène & 6 & 8 & 12 & 13 & 10 & 11 \\
Informations sur les IST/SIDA & 49 & 40 & 30 & 25 & 38 & 31 \\
Puberté & 6 & $21^{*}$ & 6 & $26^{*}$ & 6 & $24^{*}$ \\
Relation intime & 13 & 10 & 11 & 13 & 12 & 12 \\
Eviter les grossesses non désirées & 21 & $43^{*}$ & 42 & $56^{*}$ & 34 & $49^{*}$ \\
Contraception & 1 & 1 & 1 & 1,38 & 1 & 1 \\
Autres & 7 & 21 & 9 & 26 & 8 \\
\hline N & $\mathbf{5 1 3}$ & & $\mathbf{7 6 6}$ & & $\mathbf{1 2 8 0}$ \\
\hline
\end{tabular}

Conclusion, à partir des déclarations des adolescent(e)s, il y a une amélioration significative du niveau de la communication entre les parents et les adolescent(e)s. La peur de communiquer avec les parents/tuteurs sur les questions de SR ayant de plus en plus tendance à s'estomper entre les deux enquêtes et les adolescent(e)s sont plus nombreux à déclarer discuter de ces questions avec leurs parents. Tout comme le niveau de la communication, on note qu'entre les deux enquêtes des évolutions significatives ont été notées en ce qui concerne les niveaux, la qualité et la fréquence de la communication. En effet, si dans la première enquête la qualité de la communication était faible selon les adolescent(e)s, durant la deuxième enquête, on note une nette amélioration des principaux indicateurs qui ont été retenu pour rendre compte de la qualité de cette communication. Quelques disparités selon le sexe, l'age et le milieu de résidence sont cependant observées. Pour les adolescent(e)s les changements qui ont été observé en ce qui concerne la communication avec les parents sont pour beaucoup liés aux causeries auxquelles ils ont participé avec leurs parents. 
En effet, comme le déclare l'un d'eux : «En participant à ces rencontres, nous comprenons de mieux en mieux, la nécessité pour nous de nous rapprocher d'avantage de nos parents et discuter avec eux le plus souvent de toutes ces questions que nous n'osions pas aborder avec eux...Pour nous ces causeries ont été très bénéfiques en changeant nos façons de nous comporter avec nos parents »

\section{LE POINT DE VUE DES PARENTS/TUTEURS}

Pour rendre compte de la communication entre les parents et leurs enfants en matière de santé de la reproduction le point de vue des parents/tuteurs a aussi été analysé. Dans ce cadre, les questions qui leurs étaient posées permettaient d'identifier les valeurs que les parents (ou tuteurs) d'adolescent(e)s souhaitent transmettre à leur enfant et les mécanismes qu'ils mettent en place pour communiquer plus efficacement avec ces derniers sur certaines questions en rapport avec la SR.

\section{Valeurs et perceptions des parents sur le vécu des adolescent(e)s}

Les attentes des parents/tuteurs vis-à-vis des adolescent(e)s sont le plus souvent fonction du sexe des adolescent(e)s. A la question de savoir quelle est la chose la plus importante pour l'avenir des garçons environ $51 \%$ des parents/tuteurs interrogés dans l'enquête de base et $49 \%$ de ceux qui ont été interrogée dans l'enquête finale ont évoqué la réussite dans les études comme étant la chose la plus importante pour l'avenir des garçons. Le mariage n'a été évoqué que par seulement $1 \%$ des parents/tuteurs comme ayant de l'importance dans la vie des garçons.

Alors que pour les garçons les parents/tuteurs attendent généralement d'eux qu'ils réussissent dans les études et trouvent un emploi rémunéré, pour les filles par contre, il semble que les parents/tuteurs attendent généralement qu'elles réussissent dans les études et se marient. La réussite dans les études a été évoquée par $47 \%$ des pères/tuteurs et $45 \%$ des mères/tutrices comme étant la chose la plus importante pour l'avenir des filles. Le mariage a été évoqué par $25 \%$ des pères/tuteurs et $26 \%$ des mères/tutrices qui considèrent l'entrée en union est la chose la plus importante pour l'avenir des filles.

Les différences observées en ce qui concerne la perception des parents/tuteurs sur l'avenir des garçons et des filles proviennent du fait que dans la société sénégalaise les modes d'établissement social ne sont pas les même pour les garçons et pour les filles. En effet, alors que pour les garçons la réussite dans les études et l'obtention d'un emploi rémunéré conditionne en grande partie le début de la vie adulte et l'accès à certains statuts et rôles sociaux, pour les filles par contre, le mariage constitue l'étape la plus importante dans leur trajectoire d'insertion sociale. Du fait de cette situation, les attentes des parents/tuteurs vis à vis des garçons et des filles ne sont les mêmes. Les parents/tuteurs attendent des garçons se réalisent professionnellement. Pour les filles, ils attendent qu'elles terminent certes leurs études mais aussi et surtout qu'elles se marient d'où l'expression wolof «sey moy tarrou jiggen » (c'est le mariage qui fait la femme).

Les parents ont été aussi interrogés sur leurs perceptions des dangers qui guettent l'avenir des adolescent(e)s. Ainsi, à la question de savoir quels sont les dangers qu'ils redoutent le plus pour l'avenir des garçons, dans l'enquête finale, les parents/tuteurs ont mis l'accent sur la délinquance 
et les mauvaises fréquentations (27\%), sur les maladies comme les IST/SIDA (17\%), à l'avenir incertain (15\%), à l'abus de la drogue et de l'alcool (13\%).

Pour les parents hommes l'incertitude de l'avenir professionnel des garçons, les mauvaises fréquentations, les maladies comme les IST/SIDA sont les trois dangers qu'ils redoutent le plus pour les garçons. Pour les femmes par contre, les maladies comme les IST/SIDA, la drogue et l'alcool sont ce qu'elles craignent le plus pour leurs garçons. Pour les filles, les grossesses non désirées constituent le danger que les parents craignent le plus. En effet, pour $25 \%$ des parents/tuteurs (dont $17 \%$ d'hommes et $28 \%$ de femmes), la grossesse non-désirée constitue le danger qu'ils redoutent pour leurs filles. Viennent, ensuite la perversion des mœurs $(22 \%)$ puis ensuite les maladies comme les IST/SIDA (16\%).

Entre les deux enquêtes, il n'y a pas vraiment de changement sauf les mères tutrices qui parlent un peu plus des grossesses non désirées.

\section{Attitude des parents/tuteurs dans la diffusion de l'information}

L'analyse des données des deux enquêtes montre une forte proportion de parents/tuteurs qui déclarent discuter de ces questions avec leurs enfants et cela aussi bien dans l'enquête de base que dans l'enquête finale. Etant donne les niveaux élevés déclarés, il n'y a pas de différence entre les 2 enquêtes. Cette proportion est légèrement plus importante dans l'enquête finale que dans l'enquête de base (Graphique 9).

L'attitude des parents/tuteurs en matière de diffusion de l'information en direction des adolescent(e)s est fortement liée au sexe des parents. Les mères/tutrice étant plus nombreuse que les hommes à discuter des questions de SR avec leurs enfants. Ainsi, dans l'enquête de base, alors que $87 \%$ des pères/tuteurs ont déclaré avoir ce genre de discussion avec leurs enfants, parmi les mères/tutrices la proportion de celles qui déclarent avoir ce genre de discussion avec les parents/tuteurs est d'environ 97\%.

Si on tient compte du sexe de l'adolescent(e)s, les parents/tuteurs discutent plus souvent avec les filles que les garçons. Dans l'enquête finale c'est $90 \%$ des parents/tuteurs qui déclarent discuter de ces questions avec leurs filles et seulement $85 \%$ déclarent en discuter avec les garçons. 
Graphique 9: Répartition (\%) des parents/tuteurs qui communiquent avec les adolescent(e)s selon le sexe

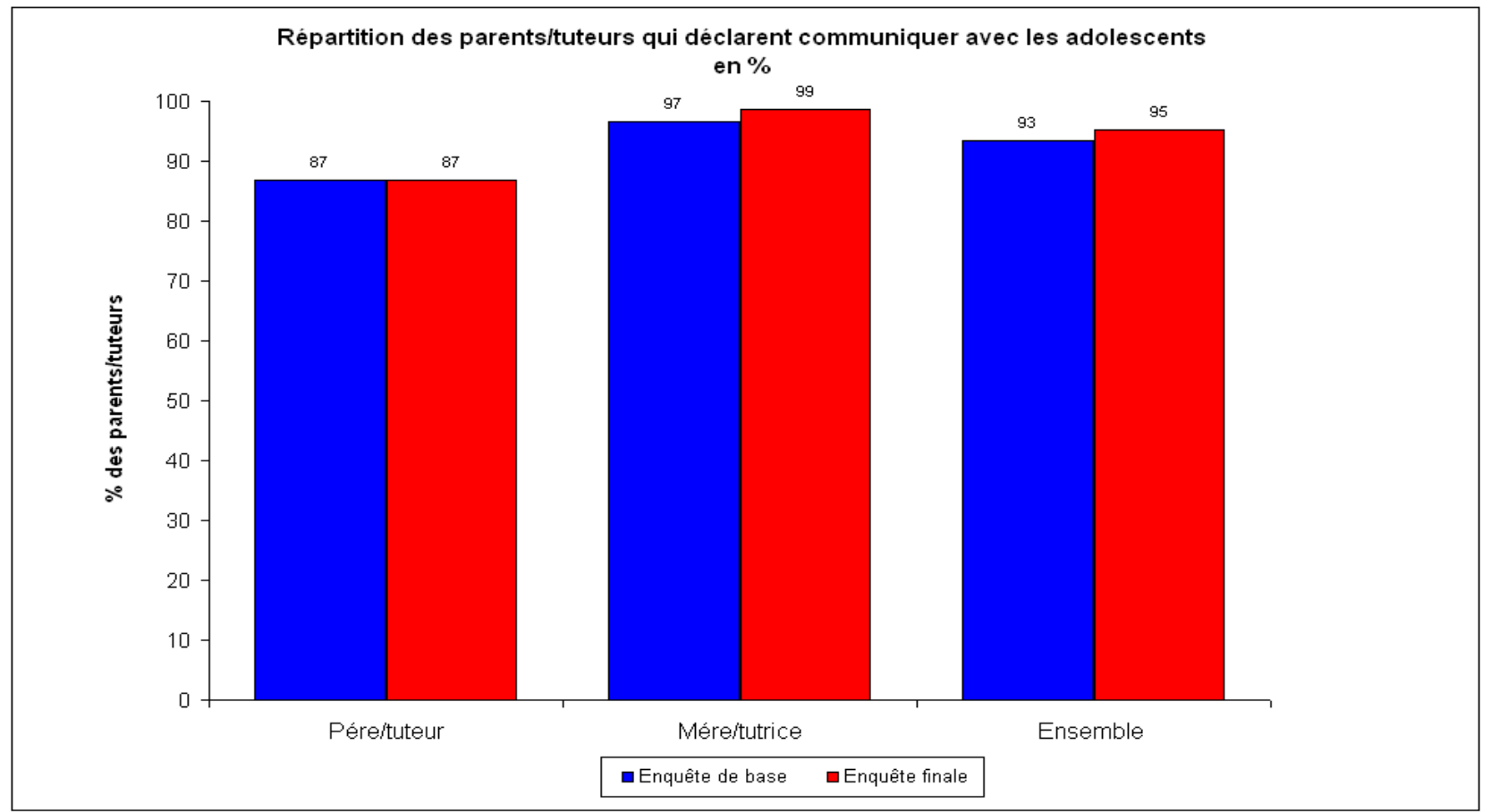

\section{PRÉfÉRENCE DES PARENTS EN MATIÈRE DE DISCUSSION}

A tous les parents/tuteurs ayant déclaré discuter des questions de SR avec leurs enfants la question suivante était ensuite posée : avec lequel de vos enfants communiquez-vous le plus? ». Par cette question, il s'agissait de voir si les parents/tuteurs avaient des préférences lorsqu'ils communiquent avec les enfants des questions de SR.

L'attitude des parents/tuteurs en ce qui concerne la communication avec leurs enfants sur les questions de SR varie beaucoup entre les deux enquêtes. La préférence des parents/tuteurs en matière de communication avec leurs enfants illustre bien cette situation. En effet, si l'enquête finale plus de la majorité des parents/tuteurs (51\%) ont déclaré ne pas tenir compte du sexe des adolescent(e)s et discutent aussi bien avec les garçons que les filles dans l'enquête de base, par contre, la proportion de ceux qui discutent aussi bien avec les garçons que les filles représente seulement $46 \%$. On remarquera, qu'il y a des variations selon le sexe de l'adolescent(e)s. Les parents/tuteurs qui déclarent discuter avec leurs filles seulement étant plus nombreux que ceux qui discutent avec leurs garçons seulement et cela aussi bien dans l'enquête de base que dans l'enquête finale (Graphique 10).

Les parents/tuteurs qui déclarent discuter des questions de SR avec leurs filles seulement étaient plus nombreux dans l'enquête de base que dans la phase d'évaluation. En effet, alors que dans la phase d'évaluation seulement $35 \%$ des parents/tuteurs déclarent discuter des questions de SR seulement avec leurs filles, dans l'enquête de base $41 \%$ des parents avaient une attitude similaire. 
Aussi bien dans l'enquête de base que dans l'enquête d'évaluation, la proportion des parents/tuteurs qui déclarent discuter de ces questions avec leurs garçons seulement est très faible. Cette proportion est d'environ 13\% dans l'enquête de base et 14\% dans l'enquête finale.

Aussi les interventions ont permis de légèrement augmenter le fait que les parents des 2 sexes discutent avec les adolescents des 2 sexes. Donc les discriminations de genre s'atténuent.

\section{Graphique 10 : Répartition (\%) des parents selon l'enfant avec lequel ils communiquent le plus, en fonction du sexe}

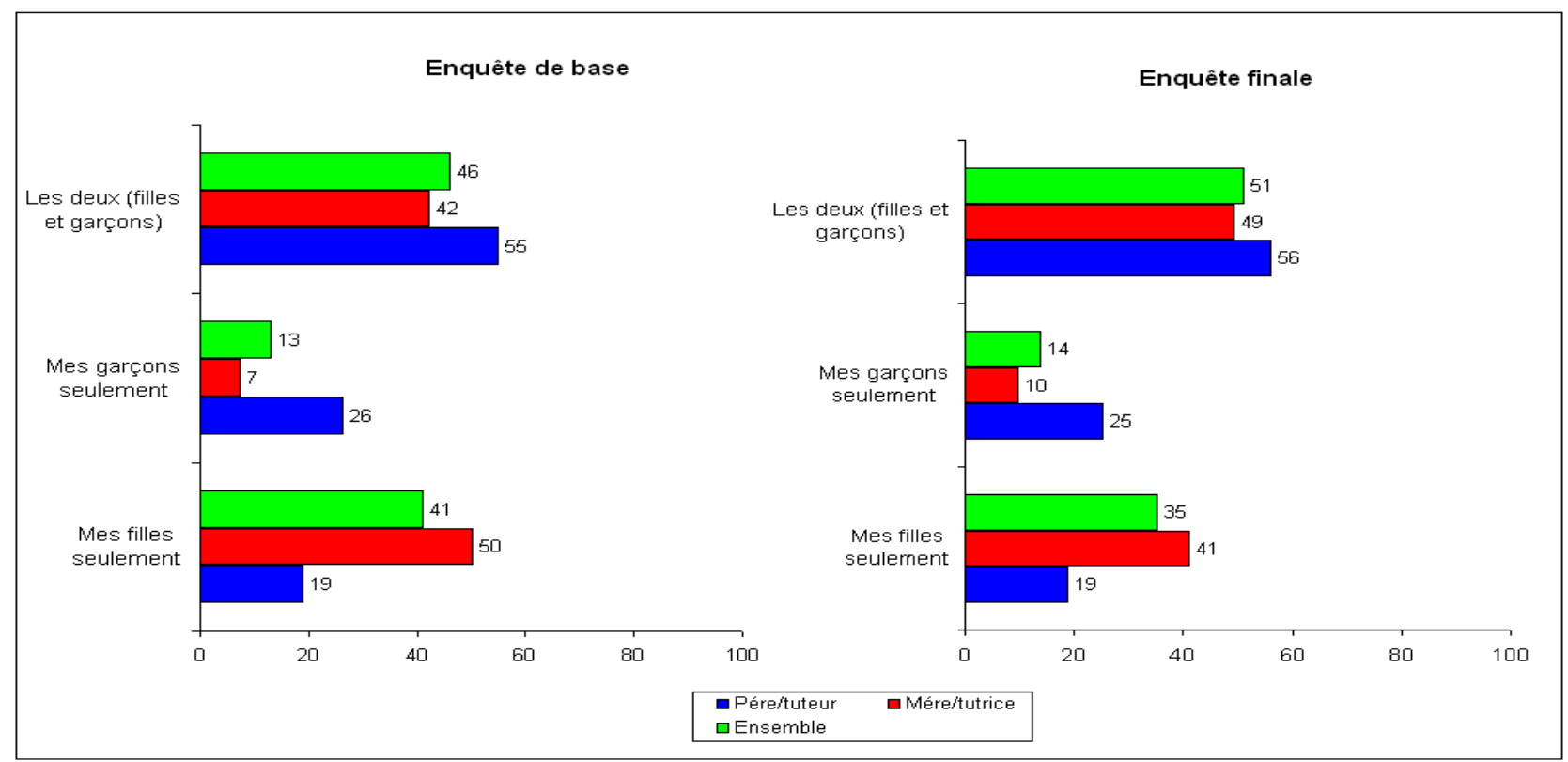

\section{CONTENU DE L'INFORMATION}

Il ressort de l'analyse des résultats que les thèmes que les parents/tuteurs déclarent discuter le plus souvent avec leurs enfants ont trait à l'abstinence/virginité, l'estime de soi, les relations avec le sexe opposé, les grossesses non désirées, les IST/SIDA. Le plus important pour eux étant de transmettre à leurs enfants certaines valeurs leur permettant d'éviter une activité sexuelle précoce.

Si l'on tient compte du sexe du parent, les femmes discutent davantage de l'abstinence/virginité, des relations avec l'autre sexe, des grossesses non désirées et de la menstruation. Ce qui peut se comprendre quant on a vu précédemment que les mères communiquent plus avec leurs filles qu'avec leurs garçons. Signalons que les IST/SIDA sont des sujets de communication tant pour les pères que pour les mères. Il en est de même pour le mariage abordé par une proportion quasi identique de pères et de mères.

En résumé, la vision des parents sur la communication avec les jeunes est beaucoup plus positive que la vision des adolescents. Etant donné les niveaux élevés rapportés dès le départ par les parents, les indicateurs de communication retenus pour les parents sont peu sensibles aux interventions. Il reste néanmoins vrai que les mères communiquent plus que les pères, et elles communiquent surtout avec leurs filles. La communication porte surtout sur le comportement, 
l'abstinence et la virginité. Les interventions ont été appréciées par les parents tels le montre ces verbatims : "Les causeries ont été organisées dans la mesure ou parents et ados ont eu la même compréhension. Actuellement, je suis en train de faire des efforts pour discuter avec mes enfants de SR. » Nous sommes satisfaits de cette intervention car depuis, il existe une facilité de communication avec nos enfants »(femmes Kaolack).

\section{COMPÉTENCE DES ADOLESCENT(E)S EN MATIÈRE DE SR}

La connaissance des adolescent(e)s en matière de santé de la reproduction peut permettre d'évaluer les programmes dont ils sont les principales cibles. Des questions ont ainsi été posées dans ce sens et sont relatives à la connaissance des risques encourus par les adolescent(e)s lors des rapports sexuels, la connaissance des IST, les méthodes de planification familiale. L'analyse des résultats des deux enquêtes montre que dans l'ensemble, la quasi-totalité des adolescent(e)s interrogés déclarent qu'il y a des risques lorsqu'un des leurs a des rapports sexuels.

Les adolescent(e)s ayant entre 10-14 ans sont les moins informés à ce sujet. Par rapport à leurs aînés, ces adolescent(e)s sont moins nombreux à connaître les risques liés à la sexualité. Au moment de l'enquête de base, $85 \%$ des 10-14 ans contre 97\% des 15-19 ans pensent en effet qu'il y a des risques liés aux rapports sexuels. A l'enquête finale il y a une progression pour les $10-14$ ans $(96 \%)$.

En tenant compte du sexe des adolescent(e)s, au tableau 8 on note qu'aussi bien dans l'enquête de base que dans l'enquête finale, les filles sont plus nombreuses que les garçons à connaître les risques associés à la sexualité. Du coté des garçons $91 \%$ de ceux qui ont été interrogés dans l'enquête de base et $97 \%$ de ceux qui ont été enquêtés dans l'enquête finale pensent qu'il y a des risques à avoir un rapport sexuel. Du coté des filles c'est respectivement $96 \%$ et $98 \%$ qui partagent ce point de vue. Dans les deux enquêtes une part importante des adolescent(e)s interrogés ( $72 \%$ dans l'enquête de base et $81 \%$ dans l'enquête finale) déclare connaître une méthode pour éviter ou retarder une grossesse.

Tableau 8: Répartition (\%) des adolescents selon leur connaissance des risques liés aux rapports sexuels et des méthodes pour éviter ou retarder une grossesse

\begin{tabular}{|c|c|c|c|c|c|c|}
\hline & \multicolumn{2}{|c|}{ Garçons } & \multicolumn{2}{|c|}{ Filles } & \multicolumn{2}{|c|}{ Ensemble } \\
\hline & $\begin{array}{l}\text { Enquête } \\
\text { de base }\end{array}$ & $\begin{array}{c}\text { Enquête } \\
\text { finale }\end{array}$ & $\begin{array}{l}\text { Enquête } \\
\text { de base }\end{array}$ & $\begin{array}{l}\text { Enquête } \\
\text { finale }\end{array}$ & $\begin{array}{c}\text { Enquête } \\
\text { de base }\end{array}$ & $\begin{array}{l}\text { Enquêt } \\
\text { e finale }\end{array}$ \\
\hline \multicolumn{7}{|l|}{ Connaissance des risques liés aux rapports sexuel } \\
\hline Connaît les risques liés aux rapports sexuels & 91 & 97 & 96 & $98^{*}$ & 94 & 98 \\
\hline$N$ & 479 & 458 & 739 & 677 & 1218 & 1135 \\
\hline \multicolumn{7}{|c|}{ Connaissance des méthodes pour éviter ou retarder une grossesse } \\
\hline $\begin{array}{l}\text { Connaît une méthode pour éviter ou retarder une } \\
\text { grossesse }\end{array}$ & 78 & $83^{*}$ & 69 & $79 *$ & 72 & $81^{*}$ \\
\hline$N$ & 407 & 390 & 529 & 547 & 936 & 937 \\
\hline
\end{tabular}


Cette connaissance est plus importante parmi les plus âgés que parmi les plus jeunes. Dans l'enquête de base $82 \%$ des 15-19 ans contre 41\% des 10-14 ans déclarent connaître des méthodes pour prévenir une grossesse. Dans l'enquête finale, ce sont respectivement $87 \%$ et $69 \%$. Il y a donc une augmentation significative pour les deux groupes d'âge.

En tenant compte du sexe des adolescent(e)s, l'écart de connaissance est moins grand entre garçons et filles. Il est de l'ordre de $9 \%$ dans l'enquête de base (78 \% pour les garçons et $69 \%$ pour les filles) et de $4 \%$ dans l'enquête finale ( $83 \%$ pour les garçons et $79 \%$ pour les filles).

Les adolescent(e)s ont également été interrogés sur leur connaissance des structures offrant des services de SR ainsi que leur opinion sur l'accès des adolescent(e)s à ces structures. Sept adolescent(e)s sur dix (72\%), ont indiqué connaître un endroit qui offre des services en matière de santé de la reproduction. Il n'y a pas de différence nette selon le sexe mais les 10-14 ans connaissent moins ces structures que les 15-19 ans (70\% contre $74 \%$ ).

\section{COMPORTEMENT SEXUEL DES ADOLESCENT(E)S}

L'analyse des données des deux enquêtes montre qu'à l'enquête de base environ 1 adolescent(e)s sur $6(16 \%)$ avait déjà eu une première expérience sexuelle au moment de l'enquête, dans la phase d'évaluation de l'étude par contre la proportion des adolescent(e)s qui déclarent avoir débuter leur vie sexuelle est d'environ 21\%. Dans les deux enquêtes, cela concerne plus les garçons que les filles, davantage les adolescent(e)s les plus âgés. En effet les adolescent(e)s ont souvent honte d'avouer qu'ils sont sexuellement actifs. L'intensification de la communication sur la sexualité durant des interventions, leur permet d'être plus à l'aise pour reconnaître leur activité sexuelle. Dans l'enquête de base $29 \%$ des garçons et $9 \%$ des filles étaient sexuellement actifs alors que dans l'enquête finale, les adolescent(e)s qui étaient sexuellement actifs étaient d'environ 33\% des garçons et 12\% de filles (Graphique 11).

\section{Graphique 11 : Répartition (\%) des adolescent(e)s selon qu'ils aient déjà eu ou non une expérience sexuelle, selon l'âge et le sexe}

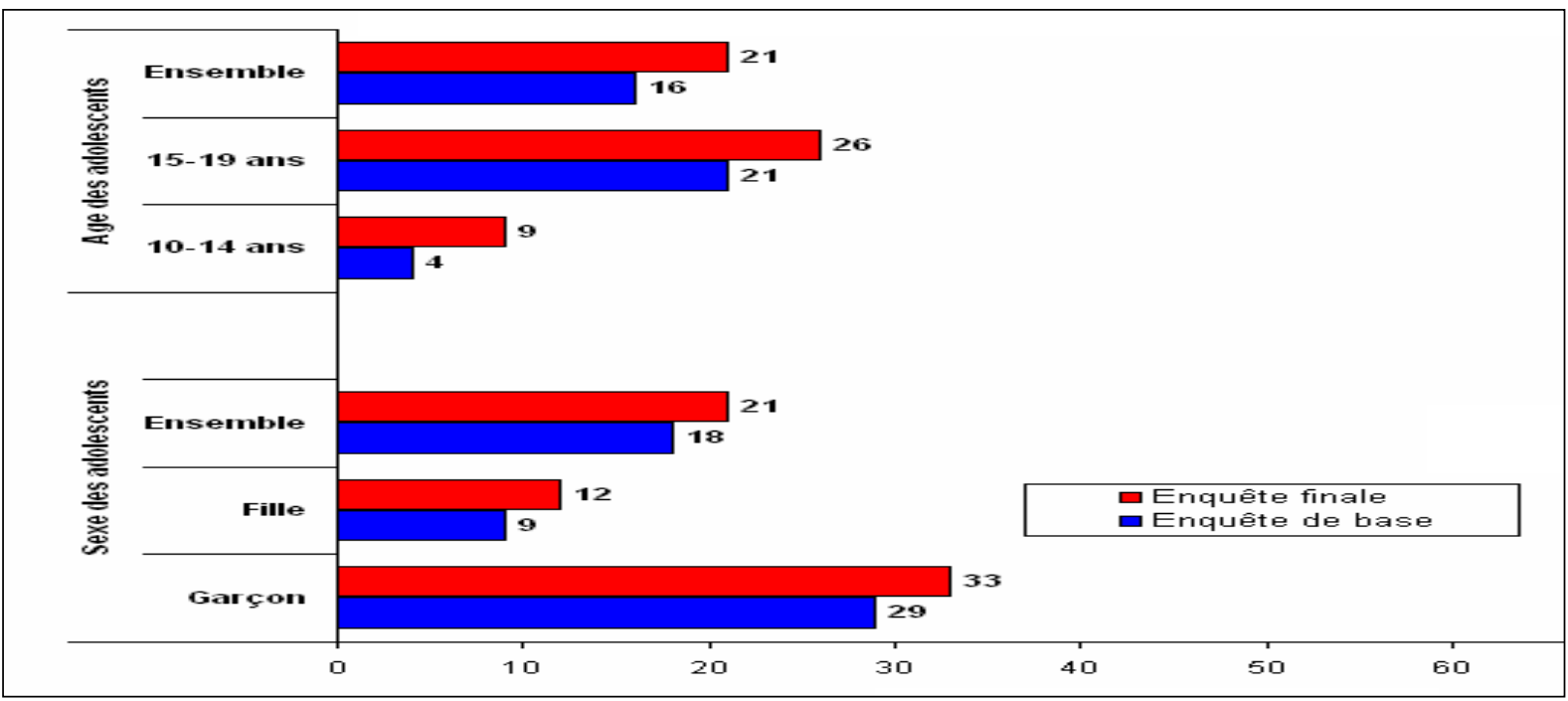


En tenant compte de l'âge, on note que les adolescent(e)s les plus âgés (15-19 ans) sont plus nombreux à avoir vécu une première expérience sexuelle que les plus jeunes. Cette situation est observée aussi bien dans l'enquête de base que dans l'enquête finale (21\% pour les 15-19 ans et $4 \%$ pour les 10-14 ans). La même situation se retrouve dans la phase finale où l'activité sexuelle concernait 26\% des adolescent(e)s ayant entre 15-19 ans et seulement 9\% des adolescent(e)s ayant entre 10-14 ans.

Cette expérience sexuelle plus fréquente parmi les garçons et les adolescent(e)s plus âgés est encore perceptible à travers les âges moyen et médian au premier rapport sexuel. En effet, L'âge d'entrée dans la vie sexuelle étant plus précoce pour les garçons que pour les filles. Les garçons ont en moyenne leur première expérience sexuelle entre 13 ans et 14 ans alors que pour les filles l'âge moyen au premier rapport sexuel se situe vers 15 ans. De même, l'âge médian au premier rapport sexuel est estimé à 14 ans pour les garçons alors qu'il est de 16 ans pour les filles. A 14 ans, la moitié des garçons sexuellement actifs avait déjà connu une première expérience sexuelle ; c'est le cas de la moitié des filles sexuellement active à 16 ans (Tableau 9).

Tableau 9: Répartition (\%) des adolescents selon l'utilisation d'une méthode de protection lors du premier rapport sexuel et lors du dernier rapport sexuel

\begin{tabular}{|c|c|c|c|c|c|c|}
\hline & \multicolumn{2}{|c|}{ Garçons } & \multicolumn{2}{|c|}{ Filles } & \multicolumn{2}{|c|}{ Ensemble } \\
\hline & $\begin{array}{c}\text { Enquêt } \\
\text { base }\end{array}$ & $\begin{array}{c}\text { Enquête } \\
\text { finale }\end{array}$ & $\begin{array}{l}\text { Enquêt } \\
\text { e base }\end{array}$ & $\begin{array}{c}\text { Enquête } \\
\text { finale }\end{array}$ & $\begin{array}{c}\text { Enquêt } \\
\text { e base }\end{array}$ & $\begin{array}{c}\text { Enquête } \\
\text { finale }\end{array}$ \\
\hline \multicolumn{7}{|l|}{ Age (en années) au premier rapport sexuel } \\
\hline Moyen & 14 & 13 & 15 & 15 & 14 & 14 \\
\hline Médian & 14 & 14 & 16 & 15 & 15 & 15 \\
\hline \multicolumn{7}{|c|}{ Utilisation d'une méthode contraceptive lors du premier rapport sexuel } \\
\hline A utilisé une méthode contraceptive lors du 1er & 31 & 33 & 13 & 19 & 25 & 28 \\
\hline$N$ & 64 & 156 & 37 & 85 & 101 & 241 \\
\hline $\begin{array}{l}\text { Utilisation d'une méthode contraceptive lors } \\
\text { du dernier rapport sexuel }\end{array}$ & & & & & & \\
\hline A utilisé une méthode contraceptive & 27 & $46^{*}$ & 15 & $31 *$ & 21 & $37 *$ \\
\hline$N$ & 64 & 156 & 37 & 85 & 101 & 241 \\
\hline \multicolumn{7}{|l|}{ Méthodes utilisées au dernier rapport sexuel } \\
\hline Préservatifs & 97 & 98 & 54 & $64 *$ & 81 & 89 \\
\hline Pilule & 0 & 0 & 5 & $45^{*}$ & 2 & 12 \\
\hline Gris-gris & 0 & 0 & 5 & 3 & 2 & 2 \\
\hline Autres méthodes & 3 & 0 & 36 & 3 & 15 & 3 \\
\hline \multicolumn{7}{|c|}{ Comportement de protection lors du dernier rapport sexuel } \\
\hline Oui & 41 & $67 *$ & 23 & 26 & 35 & $46^{*}$ \\
\hline Oui, pour éviter une grossesse & 9 & $28^{*}$ & 11 & 11 & 10 & $19^{*}$ \\
\hline Oui, pour éviter une IST/SIDA & 2 & $11^{*}$ & 1 & 0 & 2 & $5^{*}$ \\
\hline Oui, pour prévenir les deux & 30 & 28 & 10 & $15^{*}$ & 23 & 22 \\
\hline Non & 59 & $33^{*}$ & 77 & 74 & 65 & $54 *$ \\
\hline Total & 100 & 100 & 100 & 100 & 100 & 100 \\
\hline
\end{tabular}

L'examen des données des enquêtes montre que le premier rapport sexuel est le plus souvent non protégé. L'utilisation de la contraception étant très faible lors de la première expérience sexuelle. Seulement un quart $(25 \%)$ des adolescent(e)s ayant déjà eu des rapports sexuels a déclaré avoir utiliser une méthode contraceptive lors de la première expérience. Dans l'enquête finale la 
proportion des adolescent(e)s qui déclarent avoir utilisé une méthode contraceptive moderne est d'environ $28 \%$ des adolescent(e)s ayant déjà eu un premier rapport sexuel. Aussi bien dans l'enquête de base que dans l'enquête finale la première utilisation concerne d'avantage les garçons que les filles.

Les adolescent(e)s ont été également interrogés sur leur attitude de protection lors du dernier rapport sexuel. Dans ce cadre, la proportion de ceux qui déclarent avoir utilisé des méthodes contraceptives moderne lors du dernier rapport sexuellement a augmenté entre les deux enquêtes passant de $35 \%$ dans l'enquête de base à $46 \%$ dans l'enquête finale. Les méthodes utilisées au moment de l'enquête de base sont pour les garçons le préservatif $(97 \%)$ et pour les filles le préservatif (54\%), la pilule (5\%), les gris-gris (5\%) et surtout les méthodes naturelles telles le retrait. A l'enquête finale les garçons citent toujours le préservatif $(98 \%)$ et les filles le préservatif à $67 \%$ et la pilule $45 \%$. Donc un changement positif pour les filles sexuellement actives.

Les garçons rapportent en plus grand nombre le besoin de se protéger pour éviter les grossesses non désirées et prévenir les IST/SIDA.

Les interventions ont eu un léger effet sur la protection lors du dernier rapport sexuel $(p=0,002)$, surtout chez les garçons.

\section{CONCLUSIONS}

Cette recherche opérationnelle avait pour but d'évaluer la fonctionnalité et l'efficacité d'un modèle d'intervention destiné à prendre en charge les besoins d'informations des adolescent(e)s en matière de santé de la reproduction et cela à travers le renforcement et l'amélioration de la communication entre les parents et les enfants/adolescent(e)s. Les résultats qui sont issus de cette recherche permettent de tirer un certain nombre d'enseignements.

\section{En terme de faisabilité}

Les résultats de la recherche ont montré la faisabilité de mettre en place un programme qui est spécifiquement destiné à améliorer le niveau et la qualité de la communication entre les parents/tuteurs et les adolescent(e)s sur les questions de SR/VIH/SIDA et cela malgré le caractère encore sensible de cette question dans le contexte socioculturel sénégalais. Une mobilisation sociale des acteurs politiques, administratifs, religieux et communautaires a été obtenue et la disponibilité des personnes à contribuer à l'amélioration du dialogue intergénérationnel dans les familles montre une potentialité à exploiter. Un multi-partenariat entre diverses institutions a montré la faisabilité de l'approche multi-sectorielle.

Les interventions ont aussi été l'occasion d'inciter les parents et les adolescent(e)s à contribuer à la création d'espaces plus favorables pour le dialogue intergénérationnel.

La formation des acteurs de l'intervention nécessite du temps et des moyens. Elle exige que chaque type d'acteurs (relais parent, pair éducateur, modérateur et superviseur) soit formé séparément. Cependant des espaces de rencontre entre ces différents acteurs sont aussi nécessaires, sous l'égide de l'institution responsable. 
Les risques de déperdition des pairs éducateurs sont considérables, surtout en milieu urbain. Pour l'éviter, la formation d'un nombre plus important d'agents et la négociation en début de formation du contrat d'objectifs sont essentiels.

L'organisation des séances de dialogue intergénérationnelles demandent la présence d'un modérateur des discussions bien formé en capacité de négociation et de résolution des conflits est cruciale.

Ce sujet rejoint aussi les préoccupations des communautés confrontées à un environnement nouveau. Aussi l'acceptabilité de ce type d'intervention par les deux groupes a été grande.

\section{En terme d'efficacité}

La recherche montre que les interventions qui ont été menées ont contribué à l'amélioration du niveau et de la qualité de la communication entre les parents/tuteurs et les adolescent(e)s sur les questions de SR/VIH/SIDA.

La perception sur la facilité de la communication avec les parents a beaucoup évolué entre les deux enquêtes. Les adolescent(e)s étant de plus en plus nombreux à penser qu'il est facile de discuter avec leurs parents et ceci quel que soit le milieu de résidence, l'âge et le sexe de l'adolescent(e)s.

La proportion des adolescent(e)s qui déclarent discuter réellement avec les parents/tuteurs a évolué entre les deux enquêtes. Une plus grande proportion d'adolescent(e)s ont déclaré lors de l'enquête finale discuter avec leurs parents. Cette augmentation, cache toutefois des disparités selon l'âge, le genre et le milieu de résidence des adolescent(e)s. En effet, quelle que soit l'enquête, les adolescent(e)s les plus âgés et les filles sont plus nombreux à déclarer avoir des discussions avec leurs parents/tuteurs. La zone de résidence, semble aussi déterminante dans l'instauration de la relation de dialogue entre les parents et les enfants. Les adolescent(e)s issus du milieu rural sont plus nombreux que ceux du milieu urbain à déclarer avoir l'occasion de discuter librement des questions de SR avec leurs parents.

Les activités d'intervention ont aussi significativement contribué à l'amélioration de la qualité de la communication entre les parents et les adolescent(e)s en rendant cette communication plus positive et moins directive. Ainsi, dans l'ensemble, les adolescent(e)s qui déclarent discuter de ces questions ont de plus en plus l'opportunité de poser des questions à leurs parents/tuteurs et de prendre l'initiative de la discussion. Par ailleurs, les parents privilégient plus les thèmes en rapport avec la SR lorsqu'ils discutent avec leurs enfants. La fréquence de la communication a aussi significativement augmenté.

Concernant le comportement sexuel des adolescent(e)s, la recherche montre plus de franchise sur la sexualité après les interventions. Il y a une augmentation des déclarations d'activité sexuelle. En effet les adolescent(e)s ont souvent honte d'avouer qu'ils sont sexuellement actifs. L'intensification de la communication sur la sexualité durant des interventions, leur permet d'être plus l'aise pour reconnaître leur activité sexuelle. Les interventions ont aussi significativement augmenté la protection chez les adolescents sexuellement actifs. Les garçons utilisent surtout le préservatif et l'utilisation du préservatif a fortement augmenté chez les filles après les interventions, en plus de la pilule. 
Du point de vue des parents, les résultats des enquêtes ont montré que les attentes qu'ils ont envers leurs enfants en âge d'adolescence varient selon le sexe. En effet, alors que pour les garçons les parents attendent le plus souvent une réussite scolaire et un emploi, pour les filles par contre, le mariage est considéré par les parents/tuteurs comme étant plus important. Ces attitudes traduisent les survivances d'un modèle traditionnel d'entrée des jeunes dans la vie adulte qui veut que pour les garçons l'insertion dans la vie économique apparaisse comme une étape importante de la transition vers l'âge adulte alors que pour les filles le mariage passe pour être le principal mode d'établissement social et donc un passage quasi-obligé pour l'entrée des jeunes filles dans la vie adulte. Cependant les parents sont très conscients des dangers actuels que peuvent courir les adolescent(e)s.

L'attitude des parents en matière de diffusion de l'information en direction des adolescent(e)s est fortement liée au sexe des parents; Les femmes étant plus nombreuses que les hommes à discuter des questions de SR avec leurs enfants. Ces résultats ont aussi montré que les parents discutent plus souvent avec les filles que les garçons, même si cette discrimination basée sur le genre s'amenuise avec les interventions.

La communication selon les parents existe et s'appuie beaucoup sur les prêches religieuses pour que les adolescent(e)s développent des attitudes et comportements positifs. Les valeurs prônées par les parents sont surtout liées au bon comportement en société et à l'abstinence jusqu'au mariage pour les filles. Les discussions sur le VIH/SIDA avec les garçons montre une acceptation implicite de l'existence d'une sexualité chez les garçons. Les besoins d'information des parents peuvent être pris en charge à travers des séances de sensibilisation sur la SR, l'incitation à des plans d'action individuels et familiaux, et des séances de dialogue. Cependant les hommes doivent être touchés à travers d'autres canaux de communication.

Les besoins d'information des adolescent(e)s sont importants. Les parents font une sélection très importante en ne communiquant qu'avec les plus âgés et les filles. Ceci laisse les adolescents les plus jeunes assez dépourvu. C'est dans ce groupe d'âge que les besoins les plus importants sont exprimés, et que les interventions ont eu un important effet. Les parents dès le départ disent communiquer, alors que les adolescent(e)s rapportent un manque de communication important. Les parents abordent surtout les thèmes en conformité avec leurs valeurs traditionnelles. La contraception reste un sujet tabou.

L'étude sur la communication entre les adolescent(e)s et les adultes a révélé la nécessité d'intégrer cette dimension dans les programmes d'amélioration de la santé des adolescents. Les deux groupes expriment le besoin de voir s'installer cette communication. La plupart des hypothèses qui sous tendaient cette recherche ont pu être vérifiées. Le modèle testé fonctionne et a des effets sur l'amélioration de la communication. 


\section{RÉFÉRENCES}

Bruce, J. et Clark, 2004, «Les implications du mariage précoce pour les politiques de VIH/SIDA », extrait d'un article préparé pour une consultation technique de l'OMS », UNFPA et Population Council sur les Adolescent(e)ses mariées. New York, Population Council.

CEDPA/Sénégal, 2006, «Estime et confiance en soi, une arme pour les jeunes face au VIH », Curriculum de base. 182 pages.

Ceffeva and Pacific Institute for Women's Health, 1999, «Etude sur la Communication entre Parents et Enfants sur la Santé de la Reproduction », Dakar Sénégal. 72 pages.

Centre d'Etude et de Recherche sur la Population pour le Développement, 1996, «Les jeunes en danger. Résultats d'une Etude Régionale dans cinq pays d'Afrique de l'Ouest », Santé de la Reproduction au Sahel. Bamako, Mali.

Diop N.J, Dieng T., Mané B., Askew I, 2001, «Amélioration de la Santé de la reproduction des Adolescent(e)s au Sénégal : Enquête de base », Rapport d'enquête, Dakar Population Council.

Diop N. J., Dieng T., 2002, «Is parental regulation a good predictor of adolescent(e)s sexual behaviour?», Communication for the Reproductive Health Research Unit $9^{\text {th }}$ Conference, South Africa September 2002.

Diop N. J., Deme A., Bagde E., 2003, «Santé des adolescent(e)s au Sénégal : Les trois piliers d'une expérience », Population Council, Dakar-Sénégal. 45 pages

Dieng, T., Diop N. J., Bathidja H., Touré I. D., Mané B., Saumya R., Adamchak S., Wong E., Ndoye A., Sy A., Fall B., 2004, "Amélioration de la Santé de la Reproduction des Adolescent(e)s au Sénégal. Rapport final. Dakar. Population Council.

Kirby, Douglas. 2001. Emerging Answers: Research Findings on Programs to Reduce Teen Pregnancy. Washington, DC: National Campaign to Prevent Teen Pregnancy.

Locoh T., 1988 : "Structure familiale et changements sociaux" In Population et société en Afrique au sud du Sahara, édité par D. Tabutin, Paris, Harmattan, pp. 441-478

Miller, Kim S., Beth A Kotchick, Shannon Dorsey, Rex Forehand, and Anissa Y. Ham. 1998. "Family Communication About Sex: What Are Parents Saying and Are Their Adolescents Listening?" Family Planning Perspectives 30(5): 218-222 \& 235.

Moore, Kristin A., James L. Peterson, and Frank F. Furstenberg. 1986. "Parental Attitudes and the Occurrence of Early Sexual Activity." Journal of Marriage and the Family 48(4): 777-782.

Newcomer, Susan F. and J. Richard Udry. 1985. "Parent-Child Communication and Adolescent Sexual Behavior." Family Planning Perspectives 17(4): 169-174.

Pacific Institute for Women's Health. Youth Sexuality: Action Research from Burkina Faso and Senegal. Los Angeles: 2002.

Vendermermeersch C., 2002 : «Les enfants confiés de moins de 6 ans au Sénégal en 1992$1992 »$, Paris, OPCE, pp. 661-684

Whitaker, Daniel J., Kim S. Miller and Leslie F. Clark. 2000. "Reconceptualizing Adolescent Sexual Behavior: Beyond Did They or Didn't They?" Family Planning Perspectives 32(3): 111117. 